\title{
"Sonnenbarken" und "Mondscheiben“ im bronzezeitlichen Istrien? \\ Zu zwei besonderen Schalen mit verziertem Boden aus der Gradina von Monkodonja nahe Rovinj, Kroatien
}

\author{
Anja Hellmuth Kramberger
}

\begin{abstract}
Zusammenfassung
Lange Zeit war über das keramische Formenspektrum der entwickelten Frühbronzezeit und der Mittelbronzezeit der kroatischen Halbinsel Istriens nur wenig bekannt. Studien am umfangreichen Keramikmaterial aus der befestigten Bergsiedlung (Gradina) von Monkodonja an der Westküste der Halbinsel, gelegen südlich von Rovinj, haben einerseits Licht auf das variantenreiche Gefäßrepertoire geworfen, andererseits konnten über die Keramik Kontaktzonen zu den benachbarten und weiter entfernten Gebieten herausgestellt werden. Besonders deutlich, und gleichzeitig überraschend, zeichnen sich über die Keramik Verbindungen zum mittleren Donaugebiet und dem Karpatenbecken ab. In diese Richtung weisen auch einige Kleinfunde, wie beispielsweise die Funde bestimmter Nadel- und Beiltypen. Die Gradina Monkodonja und Istrien waren offensichtlich in das zentraleuropäische früh- und mittelbronzezeitliche Kommunikationsnetz eingebunden und es ist wahrscheinlich, dass der Austausch nicht nur Waren und technisches Know-how umfasste, sondern möglicherweise auch andere Kenntnisse und sogar spirituelle Vorstellungen. Hinweise darauf könnten in der Ornamentik auf der Keramik zu finden sein, deren Bedeutung sehr wahrscheinlich über die Funktion einer reinen Zier hinausging und die vielmehr als Sinnträger fungierte. Die Ornamentik auf zwei außergewöhnlichen großen Schalen von Monkodonja lässt dabei an bestimmte astrologische Kenntnisse bzw. Vorstellungen denken und wird im vorliegenden Beitrag gemeinsam mit den Verbindungen zum mittleren Donaugebiet und dem Karpatenbecken erörtert.
\end{abstract}

\section{Schlüsselbegriffe}

Früh- und Mittelbronzezeit, Istrien, Gradinen, Verzierungen auf Keramik, astrologische Kenntnisse, Himmelsscheibe von Nebra.

\footnotetext{
Abstract - 'Solar Barks' and 'Moon Discs' in Bronze Age Istria? On Two Special Bowls with Decorated Bottoms from the Gradina of Monkodonja near Rovinj, Croatia
}

For a long time, little was known about the ceramic spectrum of the developed Early Bronze Age and the Middle Bronze Age of the Croatian Istrian peninsula. Studies on the extensive ceramic material from the fortified hilltop settlement (gradina) of Monkodonja on the west coast of the peninsula, located south of Rovinj, have, on the one hand, shed light on the varied repertoire of vessels, and on the other hand, they have enabled contact zones to neighbouring and more distant regions to be highlighted. Connections to the central Danube region and the Carpathian basin are particularly clear and surprising at the same time. Some small finds also point in this direction, such as the finds of certain types of pins and hatchets. The Gradina of Monkodonja and Istria were obviously integrated into the central European Early and Middle Bronze Age communication network and it is likely that the exchange included not only goods and technical know-how, but possibly also other knowledge and even spiritual ideas. Evidence of this could be found in the ornamentation on the ceramics, the meaning of which was very likely to go beyond the function of a mere ornament and was, rather, intended to convey a meaning. The ornamentation on two extraordinary large bowls from Monkodonja suggests certain astrological knowledge or ideas and is discussed in this article together with the connections to the central Danube region and the Carpathian basin.

\section{Keywords}

Early and Middle Bronze Age, Istria, hillforts, ornaments on ceramics, astrological knowledge, Sky Disc of Nebra.

\section{Die Keramik aus der Gradina von Monkodonja und ihre Verbindungen zum mittleren Donaugebiet und dem Karpatenbecken}

Die befestigte Bergsiedlung von Monkodonja stellt den derzeit wichtigsten und am besten erforschten Fundplatz für die entwickelte Früh- und Mittelbronzezeit Istriens (Kroatien) dar. Der auf einem Hügel zwischen Rovinj und Bale gelegene Siedlungsplatz wurde erstmalig durch Boris Baćić, seinerzeit Kurator des Archäologischen Museums Istriens in Pula, zu Beginn der 50er Jahre des letzten Jahrhunderts 
durch kleinere Ausgrabungen untersucht. ${ }^{1}$ Im Jahr 1997 wurden die archäologischen Arbeiten in der Siedlung von Monkodonja als DFG-gefördertes kroatisch-deutsch-slowenisches Gemeinschaftsprojekt wieder aufgenommen und bis 2008 unter der Leitung von Kristina Mihovilić, Bernhard Hänsel und Biba Teržan fortgesetzt. ${ }^{2}$ Eine Serie von $45{ }^{14} \mathrm{C}$-Daten, die unter der Leitung von Pieter M. Grootes im Kieler Leibnitz-Labor für Altersbestimmung und Isotopenforschung anhand von tierischen und menschlichen Knochenfunden ermittelt wurden, bezeugen, dass die Siedlungsgründung um oder vor 1800 v. Chr., möglicherweise schon um 2000 v. Chr., stattgefunden hat, wenn man die Datierung der menschlichen Knochen aus den zwei Steinkistengräbern am Westtor in Betracht zieht. ${ }^{3}$ Erste Bauaktivitäten der Mauer der Hauptbefestigung fanden im 19. Jh. v. Chr. statt, die ältere Phase der Hauptnutzung der Anlage fällt in das 18.-17. Jh. v. Chr., die jüngere in das 16. bis an den Beginn des 15. Jhs. v. Chr. Nach dem chronologischen Schema Reineckes für Zentraleuropa geht es also um einen Zeitraum zwischen dem Ende der Bz A1 bis zum Übergang der Stufen Bz B1 nach Bz B2/C1.

Die intensiven Studien zur Gefäßkeramik mit detaillierten Vergleichen zum keramischen Fundspektrum der benachbarten und weiter entfernten Gebiete haben deutlich gezeigt, dass Istrien während der entwickelten Frühbronzezeit und der Mittelbronzezeit eine wichtige Kontaktzone zwischen dem adriatischen Kulturraum und Zentraleuropa darstellte. ${ }^{4}$ Einerseits umfasst das Gefäßspektrum verschiedene Formen, die als charakteristisch für Istrien, den Triester Karst und die Kvarner-Bucht zu bezeichnen sind. $\mathrm{Zu}$ nennen sind hier beispielsweise eine spezifische Form der dreieckig geformten Henkel, die an unterschiedlichen Gefäßtypen vorkommen, sog. Dreieckshenkel mit

1 Funde und Befunde aus den Grabungen von B. Baćić wurden Ende der 90er Jahre von K. Buršić-Matijašić in einer Katalogmonografie vorgelegt: BuRšIĆ-MATIJAŠIĆ 1998.

2 Die Ergebnisse wurden in den letzten Jahren durch zahlreiche Publikationen einer breiteren Öffentlichkeit vorgestellt, eine Auswahl verschiedener Aufsätze zu unterschiedlichen Themen stellen die folgenden dar: TeržAn, Minovilić, Hänsel 1999. - HäNSEl et al. 2009. - Mihovilić, HänSel, TeržAn 2011. - ZupanČič et al. 2012. - Hellmuth 2012. - Hellmuth 2014. - Die monografische Veröffentlichung der Grabungen und der Baubefunde wurde im Jahr 2015 durch die Ausgräber vorgelegt: Hänsel, Mihovilić, TeržAn 2015. - Das keramische Fundmaterial wurde 2017 durch die Autorin des vorliegenden Beitrages publiziert: Hellmuth Kramberger 2017. Im Jahr 2020 ist der dritte Band zu den Kleinfunden sowie tierischen und menschlichen Knochenfunden erschienen: HäNSEL, MiHovilić, TERŽAN 2020.

3 Hänsel, Mihovilić, TeržAn 2015, 424-452.

4 Hellmuth Kramberger 2017, 337-378. - Die Kontaktzonen finden sich auch bei anderen Artefakten wie z. B. den Bronzen aus Monkodonja reflektiert, siehe dazu die Ausführungen weiter unten.
Abschlussplatte, ${ }^{5}$ große amphorenförmige Vorratsgefäße mit x-förmigen Henkeln unterhalb des Randes, ${ }^{6}$ Dreifüße bzw. Dreifußteller ${ }^{7}$ oder sphärische Flaschen mit einer Verzierung aus Buckeln auf der Schulter. ${ }^{8}$ Andererseits lassen sich anhand der Keramik, wie erwähnt, zahlreiche Kontakte in die Nachbargebiete oder auch weit entfernte Regionen beobachten. Bemerkenswert ist dabei insbesondere ein Bezug zum mittleren Donauraum und dem Karpatenbecken, wobei sich nicht nur einzelne Stücke vergleichen lassen, sondern ein insgesamt ähnliches Gefäßspektrum mit gemeinsamen Charakteristika vorliegt. ${ }^{9}$ Besonders deutlich zeigen sich Beziehungen zwischen Istrien und dem Milieu der Aunjetitz-Kultur, genauer der Aunjetitz-Kultur im nördlichen Niederösterreich. Bekanntermaßen ist die Frühbronzezeit (Bz A1-A2 (a-b) nach der Stufengliederung Reineckes) in Niederösterreich durch das Auftreten verschiedener Gruppen gekennzeichnet, deren Keramik und Bronzen einen Bezug zur böhmischen Aunjetitz-Kultur aufweisen. ${ }^{10}$

Als die wichtigsten Gruppen der Frühbronzezeit in Niederösterreich wurden die Leithaprodersdorf-, die Wieselburger-, die Unterwölblinger-Gruppe, die MährischNiederösterreichische Gruppe der Aunjetitz-Kultur und die Böheimkirchner Gruppe der Věteřov-Kultur beschrieben. Innerhalb des Formenrepertoires der Keramik in Niederösterreich finden sich nun einerseits bestimmte Einzelstücke, die auch in Monkodonja vertreten sind und womöglich als Importe anzusprechen sind, sowie andererseits bestimmte Charakteristika in Form und Machart der Keramik, die sich allgemein parallelisieren lassen. Besonders deutlich werden die Bezüge im allgemeinen Formenspektrum bei der Feinkeramik. Charakteristisches Formengut der Feinkeramik der frühbronzezeitlichen Aunjetitz-Kultur in Niederösterreich ${ }^{11}$ sind Krüge und Becher mit kugeligem Körper und abgesetztem Hals, wie sie

5 Hellmuth Kramberger 2017, 244-248.

6 Hellmuth Kramberger 2017, 176-179, 202-205, 240-242.

7 Hellmuth Kramberger 2017, 215-220. - Hierbei ist zu bemerken, dass es jedoch um eine Form geht, die aller Wahrscheinlichkeit nach auf ostmediterrane Vorbilder zurückgeht: HänsEL, TERŽAN 2000, 179. - Mihovilić 2001, 47-48. - Hänsel, Mihovilić, TeržAN $2015,503$.

8 Hellmuth Kramberger 2017, 116-124.

9 Hellmuth Kramberger 2017, 367-378, 409.

10 Neugebauer 1987, 19. - Bertemes 1989, 164-167, 167 und Abb. 73. - Neugebauer 1994, 49-71. - Lauermann 2003. - Zur böhmischen Aunjetitz-Kultur bspw.: Moucha 1963. - BARTELHeim 1998. - Hinz 2009. - Zur nördlichen Aunjetitz-Kultur bspw.: ZICH 1996.

11 Lauermann 2003, 587-591. - Für die Aunjetitz-Kultur in Böhmen siehe die Typengliederung der Krüge und Becher bei BARTELHEIM 1998, 23-28. 


ANDERE FUNDORTE

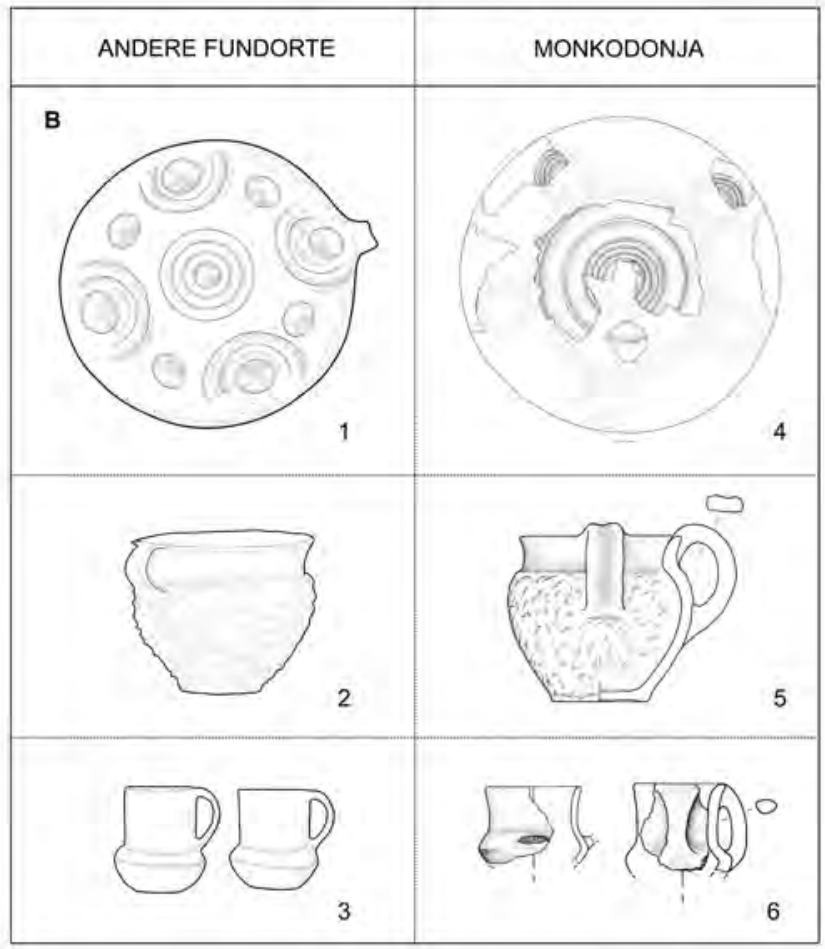

Abb. 1. Vergleiche zwischen keramischen Formen aus Monkodonja und dem Donau-Karpatenbecken (zusammengestellt nach HeLLmuTH Kramberger 2017, Abb. 273, 275).

beispielsweise aus dem Gräberfeld von Gemeinlebarn bekannt sind (Abb. 1/A4-5). ${ }^{12}$ Sie finden sich in zahlreichen Varianten von unterschiedlichen Proportionen in der Relation von Randdurchmesser zum Bauchmaximum, der Länge und Stärke der Auswärtsrichtung des unverdickten, stets abgesetzten Randes sowie mit und ohne Henkel. ${ }^{13}$ Derartige Krüge, Becher und kleine Töpfe mit abgesetztem, unverdicktem Hals und kugeligem Gefäßkörper bzw. Unterteil

12 Bertemes 1989, Taf. 25/9 (vgl. Hellmuth Kramberger 2017, z. B. Taf. 51/6); Taf. 12 (vgl. Hellmuth Kramberger 2017, z. B. Taf. 107/3); Taf. 26/9 (vgl. Hellmuth Kramberger 2017, z. B. Taf. 136/7); Taf. 29/Grab 74,4 (vgl. Hellmuth Kramberger 2017, z. B. Taf. 51/6); Taf. 33/Grab 96,3; Taf. 34/Grab 111,6; Taf. 39/ Grab 134,2; Taf. 45/6/Grab 159,6; Taf. 59/Grab 227,15 und 237,2; Taf. 62/Grab 251,1 und 257,1. - Andere Fundorte sind beispielsweise Franzhausen (Neugebauer, Neugebauer 1997, Taf. 462/5b. - NeuGeBauer, Blesl 1998, 414 und Abb. 115/2), Eggenburg (LauermanN 2003, 40 und Abb. 20/2), Großmugl (Lauermann 2003, 92, Nr. 60) oder Großweikersdorf (Lauermann 2003, 106, Nr. 8).

13 Wie auch in Monkodonja handelt es sich um dunkle, gut geglättete oder polierte Keramik, siehe bspw. ZICH 2004, 136. bilden nun auch in Monkodonja das charakteristische Formenspektrum der Feinkeramik bzw. des Trinkservices. ${ }^{14} \mathrm{Zu}$ denjenigen Stücken aus Monkodonja, die mit sehr großer Wahrscheinlichkeit einen Import darstellen und damit einen deutlichen Bezugspunkt in den niederösterreichischen Raum bilden, gehört ein kleiner scheibenförmigen Deckel mit leicht nach unten gewölbter Unterseite (Abb. 1/A6). ${ }^{15}$ Solche Deckel stellen in Böheimkirchen eine ausgesprochen charakteristische Form dar (Abb. 1/A1). ${ }^{16}$ Weitere Formen, welche Monkodonja mit Böheimkirchen verbinden, sind die Gefäße mit flächendeckender Verzierung aus kleinen Knöpfen, ${ }^{17}$ Dornengefäße bzw. Gefäße mit Zapfen

14 Hellmuth Kramberger 2017, Abb. 66/ IVa-c; 274/f, h.

15 Hellmuth Kramberger 2017, Taf. 68/2, Abb. 273/b.

16 Neugebauer 1977, 71 und Abb. 9G/10-11; 154 und Taf. 28/8; 183 und Taf. 64/1; 187 und Taf. 69/2; 201 und Taf. 87/8.

17 Hellmuth Kramberger 2017, Taf. 25/2.5; 31/2; 38/3; 94/6; 139/1; Abb. 231/a-c; 273/e, f1-2. - Vgl. mit Neugebauer 1977, 143 und Taf. 15/1; 155 und Taf. 30/4; 176 und Taf. 55/2; 194 und Taf. 77/11. 
an der Innenwand ${ }^{18}$ und „baguetteförmige“ plastische Leisten. ${ }^{19}$ Dabei gehört die Verzierung der Gefäßoberfläche mit kleinen Knöpfen zu den Charakteristika der früh- bis mittelbronzezeitlichen Fundplätze im mittleren Donaugebiet wie der Aunjetitz-Kultur im nördlichen Niederösterreich, ${ }^{20}$ der Hatvan-Kultur ${ }^{21}$ oder der Věteřov-Kultur ${ }^{22}$ (Abb. 1/A3, 8). In Bezug auf das niederösterreichische Material kann auch auf eine Parallele zwischen Funden aus Monkodonja und Gefäßen aus einem frühbronzezeitlichen Fundplatz bei Gollnsdorf/Leobersdorf hingewiesen werden. ${ }^{23}$ In Gollnsdorf/Leobersdorf wurden Randfragmente eines oder mehrerer großer Töpfe mit vollplastischem $\mathrm{x}$-förmigem Henkel („Pseudo-x-Henkel“) und horizontaler plastischer Leiste gefunden, ${ }^{24}$ die sehr deutlich an einen Pithos und einen kleinen Topf mit identischer Randgestaltung aus Monkodonja erinnern. ${ }^{25}$ Als „Bindeglieder“ zwischen Niederösterreich und dem Istrischen Karstgebiet inklusive Südwestslowenien, welches in den Kulturkreis der Gradinen eingebunden war, $^{26}$ können bestimmte keramische Einzelfunde angesprochen werden, die im Gebiet der österreichischen Steiermark gefunden wurden. Besonders deutlich wird dies in Form von Gefäßfragmenten, die frappant an den Typ der amphorenförmigen Töpfe und Pithoi mit x-Henkeln aus Monkodonja erinnern (Abb. 2a). Zu denken wäre hier zunächst an ein Gefäß aus Vorwald bei Wald am Schoberpass. $^{27}$ Interessant ist jedoch auch ein Gefäßrand mit Henkel, der jüngst von Georg Tiefengraber veröffentlicht wurde und der aus dem umfangreichen Keramikmaterial vom Wildoner Schlossberg stammt, eine der prominentesten Fundstellen im südlichen Grazer Becken an der Mur,

18 Hellmuth Kramberger 2017, Taf. 113/6. - Zwei weitere Dornengefäße aus den Altgrabungen auf Monkodonja: BuRšśćMatijašıć 1998, 61, 63 und Taf. 13/234.236. - Vgl. Neugebauer 1977, 153 und Taf. 26/1; 175 und Taf. 54/9; 181 und Taf. 61/5. LochNer 2012, 199-200. - Hellmuth 2014.

19 Hellmuth Kramberger 2017, Taf. 76/1. - Vgl. Neugebauer 1977, 143 und Taf. 15/12; 171 und Taf. 49/2; 174 und Taf. 52/7; 53/1; 177 und Taf. 57/2; 186 und Taf. 68/14; 198 und Taf. 83/5; 199 und Taf. 84/11-12; 201 und Taf. 87/6; 196 und Taf. 80/5.

20 Lauermann 2003, 595, 120 und Abb. 53/39; 134 und Abb. 60/20; 397, Nr. 7; 401 und Abb. 184/7.13-14.

21 Kalicz 1968, Taf. 60/6; 61/5. - Vgl. Stuchlík 2003, 448.

22 Stuchlík 2003, 448, 464 und Abb. 7/13-14. - Neugebauer 1977, 64, 65 und Abb. 7D2; 143 und Taf. 15/1; 155 und Taf. 30/4; 176 und Taf. 55/2; 194 und Taf. 77/11.

23 J.-W. Neugebauer ordnete die Funde aus Gollnsdorf/Leobersdorf dem Beginn der Bronzezeit zu (BZ A1a): Neugebauer 1976, 51. 24 Neugebauer 1976, 72 und Abb. 3/2-3.

25 Hellmuth Kramberger 2017, Taf. 41/8; 152/2; Abb. 273/h.

26 Vgl. dazu Hellmuth Kramberger 2017, 353-365.

27 Schamberger 2007, Taf. 6/24. - Vgl. Hellmuth Kramberger 2017, Abb. 171/a. gelegen an einem strategisch bedeutsamen und verkehrsgeografisch wichtigen Schnittpunkt des Südostalpenraumes (Abb. 2b). ${ }^{28}$ Formal betrachtet entspricht das besagte Gefäßfragment vom Wildoner Schlossberg den Randformen bzw. Gefäßoberteilen der amphorenförmigen Töpfe und Pithoi Monkodonjas und Istriens bis ins Detail. ${ }^{29}$ Von besonderer Bedeutung ist außerdem, dass Tiefengraber bemerkt, dass die Faktur des Stückes deutlich vom übrigen Bestand der Keramik vom Wildoner Schlossberg abweicht. ${ }^{30}$ Er interpretiert das Randfragment als „kümmerlichen Rest eines aus dem oberadriatischen Küstengebiet importierten Gefäßes [...], wobei dieses selbst wohl eher als Transportmedium bzw. Verpackung fungiert haben wird und nicht die eigentliche Handelsware dargestellt haben dürfte". ${ }^{31}$ Um welche Art der Handelsware es sich gehandelt haben dürfte, bleibt letztendlich unbekannt, doch es ist durchaus möglich, dass die amphorenförmigen Töpfe und Pithoi in Istrien zur Aufbewahrung von Wein gedient haben. ${ }^{32}$

Dass Istrien in das früh- und mittelbronzezeitliche Handels- und Kommunikationsnetz mit Zentraleuropa und insbesondere mit dem mittleren Donauraum und dem Karpatenbecken eingebunden war, wird auch durch andere Funde aus der Gradina von Monkodonja deutlich. ${ }^{33} \mathrm{Zu}$ nennen ist im Zusammenhang mit den Bronzefunden ${ }^{34}$ eine Hülsenkopfnadel mit großer rhombischer Kopfplatte und schwach tordiertem Schaft, die im Bereich des Westtores in der Grabungsfläche Sonda V unter der Schuttlage der jüngeren Befestigung gefunden wurde. ${ }^{35}$ Die besten Vergleiche für die Nadel aus Monkodonja finden sich laut Hänsel und Teržan im mittleren Donauraum, wo möglicherweise auch ihre Herkunft zu suchen ist. ${ }^{36}$ Eine Hülsenkopfnadel mit

28 Tiefengraber 2018, 222 und Abb. 261/19; 228-229.

29 Vgl. Hellmuth Kramberger 2017, 178, 203-205. - Es geht um die Variante a2, einen dreieckig verdickten Rand mit Rille auf der Oberseite: Hellmuth Kramberger 2017, 177, 178 und Abb. 146/a2. 30 Tiefengraber 2018, 229.

31 Tiefengraber 2018, 229.

32 Hellmuth 2014, 71. - Ein ähnlicher Gefäßrand liegt auch aus dem bislang unpublizierten multiperiodischen Fundplatz Obrežje an der Save nahe der Grenze zwischen Slowenien und Kroatien, ca. $20 \mathrm{~km}$ von Zagreb entfernt, vor. Die Information verdanke ich B. Kramberger.

33 Hänsel, Mihovilić, Teržan 2015, 503. - Hänsel, Mihovilić, TERŽAN 2020.

34 Die chemischen Analysen an verschiedenen Bronzen aus Monkodonja haben gezeigt, dass für die Metalllegierungen verschiedene Sorten an Rohkupfer verwendet wurden und somit aus unterschiedlichen Erzquellen stammen: URANKAR 2020. Eine Bestimmung der Provenienz des Rohkupfers war dabei jedoch nicht möglich. 35 Hänsel 2002. - Hänsel, Mihovilić, TeržAn 2015, 449 und Abb. 112. - HÄNSEL, TERŽAn 2020, 166 und Abb. 5; 205-208 und Abb. 22-23. 36 Hänsel, TeržAn 2020, 207 und Abb. 22. 

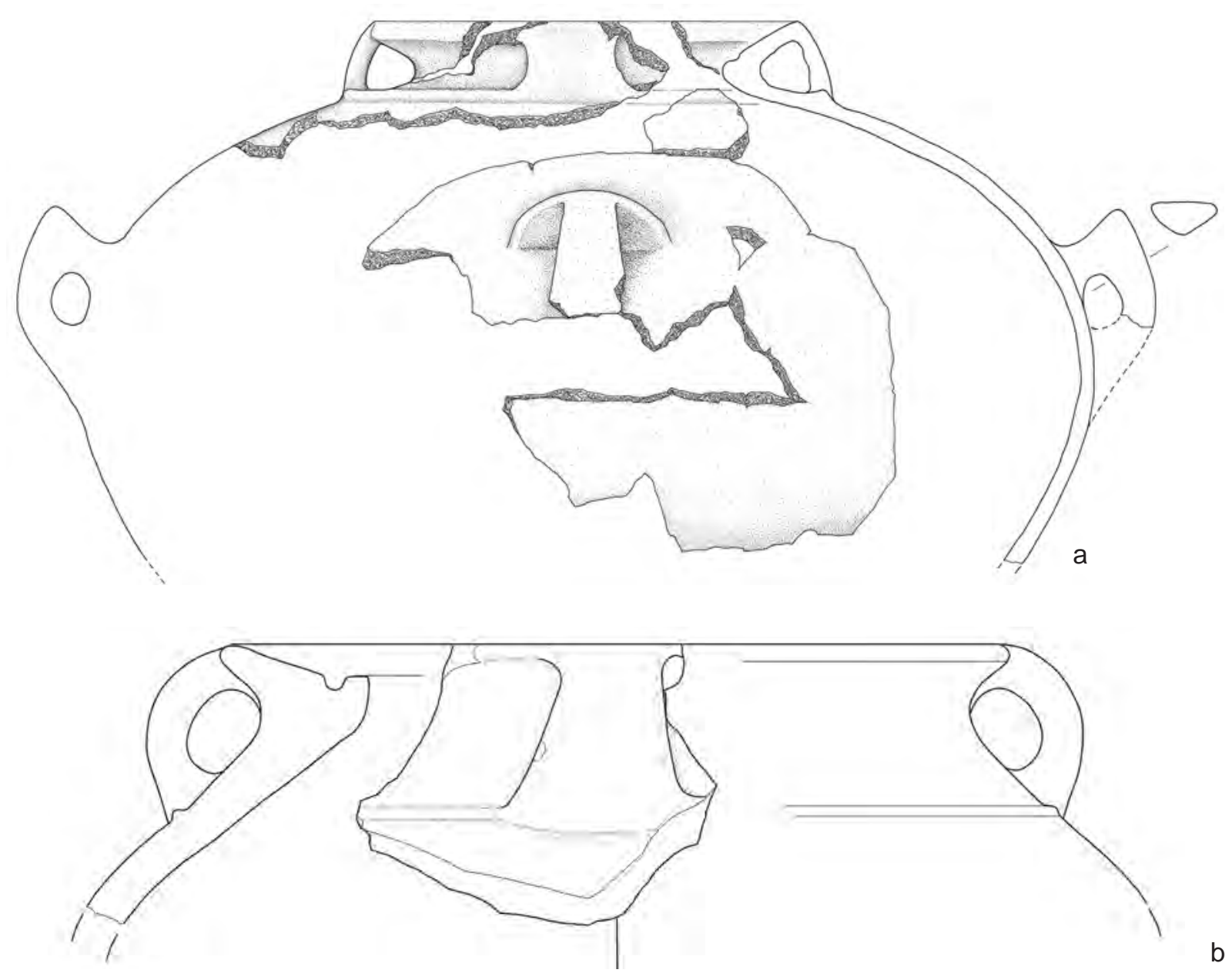

Abb. 2. Amphorenförmige Vorratsgefäße aus Istrien und der Steiermark. - a. Monkodonja (nach Hellmuth Kramberger 2017, Taf. 37/3). b. Wildon, Schlossberg (nach Tiefengraber 2018, Abb. 261/19).

glattem Schaft kann auch aus dem frühbronzezeitlichen Gräberfeld von Gemeinlebarn in Niederösterreich angeführt werden, und zwar im Grab 93. ${ }^{37}$ Dieses Grab ist insofern interessant, da es auch eine Schüssel mit rechteckig nach außen verdicktem Rand und Bandhenkel mit runder Seitenansicht enthielt, die deutlich an ein Gefäß aus Monkodonja erinnert. ${ }^{38}$ In das Karpatenbecken weisen auch das Fragment einer Sichelnadel aus Monkodonja ${ }^{39}$ und ein Blechtutulus mit breiter Krempe, der mit zwei konzentrischen Kreisen mit Hohlbuckelchen verziert ist. ${ }^{40} \mathrm{Im}$ ostösterreichischen Raum bis nach Passau, Mähren und die Slowakei finden sich dieParallelen für ein Lappenbeil mit kleinen, mittelständigen

37 Bertemes 1989, Taf. 5/unten Mitte, Taf. 32/Grab 93.

38 Hellmuth Kramberger 2017, 370, 371 und Abb. 272/a, d.

39 Hellmuth Kramberger 2017, 208 und Taf. 6/2.

40 Hellmuth Kramberger 2017, 216 und Taf. 7/13.
Lappen aus Monkodonja, das der Variante Unterradl des Typs Gmunden nahesteht. ${ }^{41}$

Auch das Vorkommen von Brotlaibidolen in Monkodonja ist als weiteres, deutliches Zeichen der Einbindung Monkodonjas bzw. Istriens in den Kommunikationsraum zwischen mittlerem Donaugebiet und Karpatenbecken, dem zirkumalpinen Raum mit Zentrum im Bereich der Polada-Kultur um den Gardasee in Norditalien sowie dem Verbreitungsgebiet der Aunjetitz-Kultur zu benennen. ${ }^{42}$ Das Vorkommen dieser „enigmatischen Täfelchen“ ist mit einer Reihe früh- und mittelbronzezeitlicher Kulturen bzw. kultureller Gruppen verknüpft, wie neben der

41 Hänsel, TeržAn 2020, 184 und Taf. 2/1, Abb. 2. - Vgl. Mayer 1977, 128-130 und Taf. 33/482-483 u. a.

42 Mihovilić, Hänsel, Teržan 2011. - Mihovilić, Hänsel, TeržAn 2017. - MihoviLić 2020. 

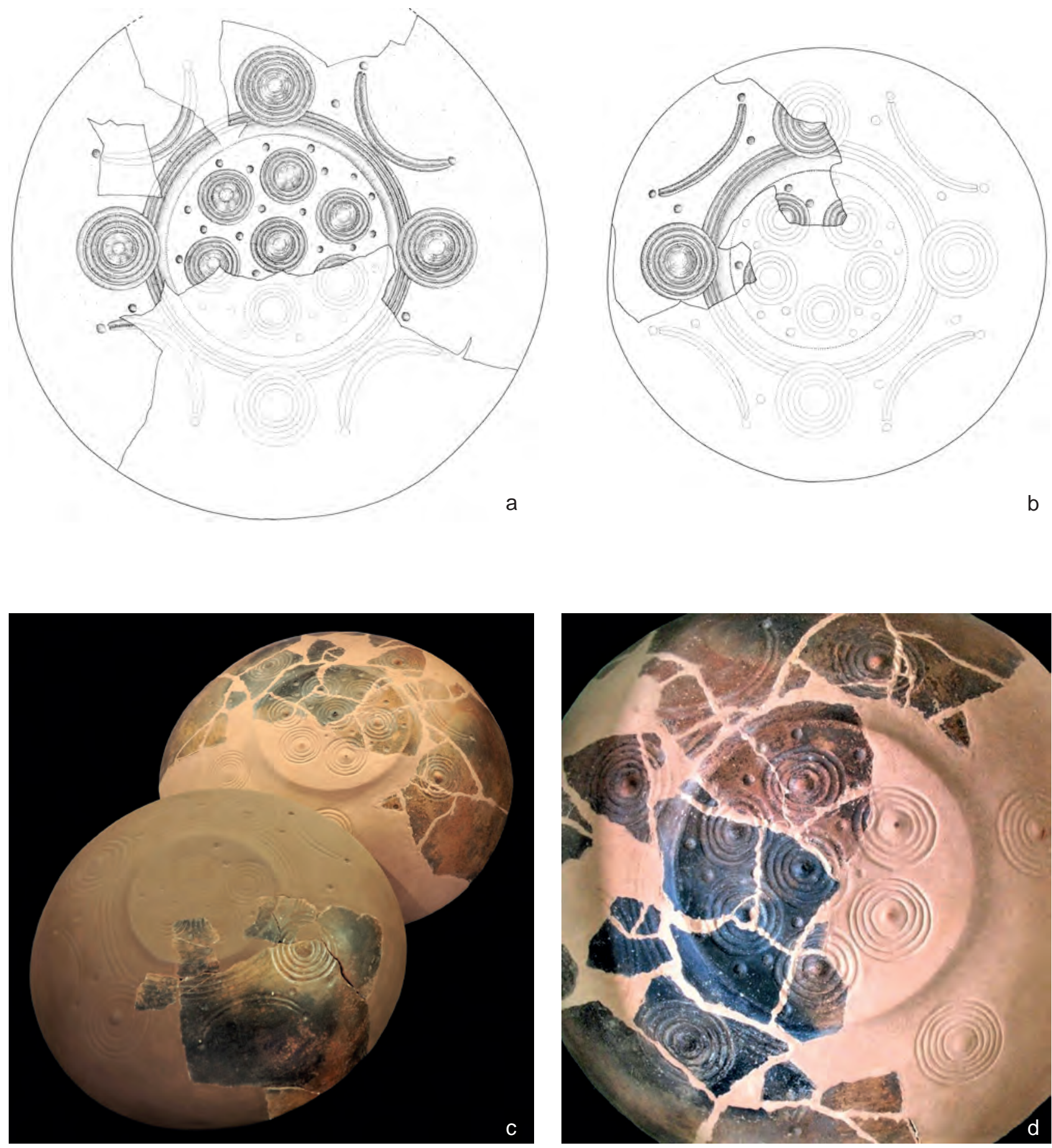

Abb. 3. Große Schalen mit verzierter Unterseite aus Monkodonja, Istrien (a-b. nach Hellmuth Kramberger 2017, Taf. 45-46. c. Foto: D. Matošević. - d. Foto: K. Mihovilić, @ Archäologisches Museum Istriens/AMI Pula). 
genannten Polada-Kultur Norditaliens oder der AunjetitzKultur etwa auch die Füzesabony-Kultur Ungarns oder die Böheimkirchner Gruppe Niederösterreichs. ${ }^{43}$ Es wird nun demzufolge kein Zufall sein, dass sich in der Keramik Monkodonjas deutliche Einflüsse aus ebendiesen Gebieten fassen lassen, einerseits in Form vergleichbarerer Merkmale im Formenspektrum, andererseits in Form von mutmaßlichen Importen. ${ }^{44}$

Der Austausch innerhalb dieses weitgespannten Kommunikationsnetzes dürfte jedoch nicht nur den Austausch von Waren und handwerklichem Know-how umfasst haben, sondern wahrscheinlich auch von intellektuellem und religiösem Wissen und Vorstellungen. Vor diesem Hintergrund soll im Folgenden auf einige besondere keramische Funde aus Monkodonja eingegangen werden, deren markante Verzierungen möglicherweise auf bestimmte astrologische Kenntnisse und womöglich damit verknüpfte spirituelle Vorstellungen hindeuten könnten.

\section{Schalen mit verzierter Bodenunterseite aus Monkodonja}

Gegenstand der Betrachtungen bilden zwei fragmentarisch erhaltene, große Schalen von gut $50 \mathrm{~cm}$ Randdurchmesser (Abb. $3 a-d) .{ }^{45}$ Sie tragen auf der Außenseite und der Bodenunterseite eine komplexe Verzierung bestehend aus umlaufenden konzentrischen Kanneluren, eingetieften Punkten, unterschiedlich großen konzentrischen Kreisen mit kleinen Buckeln („Tutuli“46) in der Mitte sowie bogenförmigen Objekten.

Bei den besagten Schalen handelt es sich um Funde, die im Grabungsabschnitt der Sonda VI zutage gekommen sind, also jenem Eckbereich südlich von der Torgasse des Westtores der Hauptbefestigung von Monkodonja. ${ }^{47}$ Von besonderer Bedeutung ist, dass sich im Eckturm der Toranlage ein Steinkistengrab (Steinkistengrab B) fand, ${ }^{48}$ ein weiteres Steinkistengrab (Steinkistengrab A) wurde auch im Bereich der nordwestlichen Flanke des Westtores entdeckt. ${ }^{49}$ Beide Steinkisten wurden, so bezeugen es die ${ }^{14} \mathrm{C}$-Datierungen, die anhand menschlicher Knochen gewonnen wurden, über mehrere Jahrhunderte belegt, von

43 Z. B. Piccoli, Laffranchini 2011, 21.

44 Eine Gesamtdarstellung: Hellmuth Kramberger 2017, 346378 .

45 Hellmuth Kramberger 2017, Katalogteil, 38 und Taf. 45/2; 46.

46 Im Fall der Keramik aus Monkodonja wurde der Bezeichnung „Tutulus“ den Vorrang gegeben (Hellmuth Kramberger 2017, 267), da die - insbesondere an Flaschen vorkommenden - Ausbuchtungen oft hohl gearbeitet sind und eine spitze kegelförmige Gestalt aufweisen. Möglicherweise imitieren sie Vorbilder aus Metall.

47 Hänsel, Mihovilić, TeržAn 2015, 71, 119-121.

48 Hänsel, Mihovilić, Teržan 2015, 211-224.

49 Hänsel, Mihovilić, Teržan 2015, 199-211. ca. 2000 v. Chr. bis in die zweite Hälfte des 17. Jhs. v. Chr. ${ }^{50}$ Die beiden Gräber wurden in den Jahrhunderten nach ihrer Errichtung unterschiedlich behandelt. Nach dem Ende der Belegung wurde das Grab A durch Überbauungen der Sicht entzogen, d. h. es wurde zwar einerseits versteckt, doch gleichzeitig bewahrte man auch das Andenken an die darin Bestatteten durch seinen Erhalt. Im Siedlungsinneren bzw. jenem inneren Bereich, der an den Eckturm der Toranlage mit dem Steinkistengrab B angrenzt, wurden abgesehen von den beiden relevanten Schalen auch größere Mengen an Gefäßkeramik geborgen. ${ }^{51}$ Unter diesen fällt insbesondere die Anzahl weiterer Sonderformen der Schalen mit verziertem Boden auf, die nur in diesem Bereich dokumentiert werden konnten. ${ }^{52}$ Genannt werden können darunter auch mindestens fünf identische Schalen mit unterschiedlich breiten konzentrischen Kanneluren an der Bodenunterseite, in deren Mitte ein Buckel („Tutulus“) sitzt; eine Punktreihe schließt jeweils die äußerste konzentrische Kannelur ab (Abb. 4). ${ }^{53}$

Die fünf kleinen identischen Schalen weisen eine gut geglättete, ursprünglich womöglich sogar polierte ${ }^{54}$ Oberfläche auf und wurden im Oxidationsbrand hergestellt, ihre Oberflächen auf der Innen- und Außenseite sowie im Kern sind von einem kräftigen beigen Farbton mit leicht oranger

50 Hänsel, Mihovilić, Teržan 2015, 431, 433-434, 439 und Abb. 320; 449, 509.

51 Hellmuth Kramberger 2017, Katalogteil, 35-42 und Taf. 3956.

52 Unter 7420 im Detail analysierten Gefäßen und Gefäßfragmenten aus Monkodonja konnten 143 Schalen mit verziertem Boden (2 \%) identifiziert werden: Hellmuth Kramberger 2017, 309-310 und Abb. 247. Dabei kann jedoch noch einmal hervorgehoben werden, dass sich in jenem, dem Eckbau des Westtores mit dem Steinkistengrab B vorgelagerten Bereich eine auffällige Zahl an Varianten von Schalen mit verziertem Boden fand, die sonst in keinem anderen untersuchten Areal der Siedlung vorkommen, also etwas Besonderes darstellen.

53 Hellmuth Kramberger 2017, 38, 138-139 und Taf. 44-45/1; 153/13. - Abgesehen von den benannten fünf Schalen sowie den beiden Schalen, die Gegenstand des vorliegenden Aufsatzes sind, fanden sich auch weitere Fragmente von Schalen mit verziertem Boden, die jedoch allesamt sehr fragmentarisch erhalten sind.

54 Dies lässt sich jedoch auf Grund des Erhaltungszustandes nicht mehr eindeutig bestimmen, die Oberflächen sind erodiert und zum Teil von Ablagerungen überzogen, was auf der Abb. 4 speziell auf der linken Hälfte der Schale erkennbar ist. Unter den bestimmbaren Stücken nehmen polierte Oberflächen 19 \% ein (Hellmuth KramberGER 2017, 41), sie zeichnen sich durch einen metallischen Glanz aus: Hellmuth Kramberger 2017, 44 und Abb. 7. Es ist anzunehmen, dass die Gefäßoberflächen zunächst mit einem harten Gegenstand geglättet wurden (z. T. sind deutliche Glättspuren an der Keramik erkennbar), im Anschluss wurden diese mit Hilfe eines weichen $\mathrm{Ob}$ jekts, z. B. Tuch, poliert, bis ein hochgradiger Glanz erzielt war: vgl. z. B. SCHNEIDER 1989, 13. 


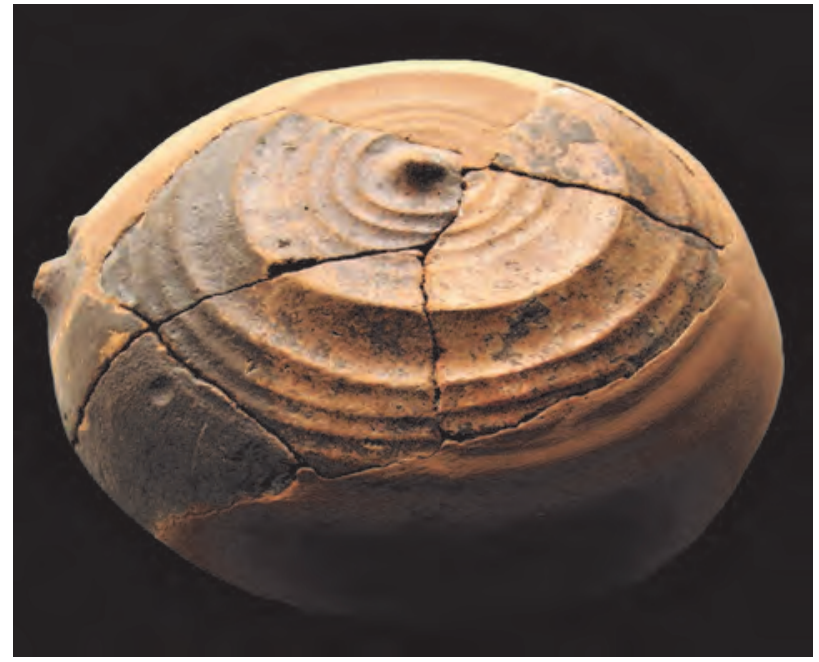

Abb. 4. Kleine Schale mit verzierter Unterseite aus Monkodonja (Foto: K. Mihovilić, ( ) Archäologisches Museum Istriens/AMI Pula).

Nuance und es ist gut vorstellbar, dass versucht wurde, den Schalen das Aussehen von Metallgefäßen - Bronzegefäßen - zu geben. ${ }^{55}$ Auf Grund der Buckel („Tutuli“) auf der Mitte der Bodenunterseite erscheint es wahrscheinlich, dass die Schalen als Deckschalen benutzt wurden. ${ }^{56}$ Die Bodenunterseite der Schalen wurde somit zur Schauseite. Etwas Ähnliches ist nun auch für die beiden großen Schalen anzunehmen, die im Zentrum der vorliegenden Betrachtung stehen. Sie zeigen eine gut geglättete Oberfläche auf der Innen- und Außenseite und waren im Oxidationsbrand hergestellt, wobei ihre Farbgebung stark uneinheitlich ist und zwischen verschiedenen Beige-, Braun- und Orangenuancen changiert. Während sich von der einen, etwas kleineren, kalottenförmigen Schale mit steilerem Wandprofil nur ein knappes Viertel erhalten hat (Abb. 3b), ${ }^{57}$ sind von der zweiten, geringfügig größeren Schale mit flacherem Wandprofil ca. zwei Drittel vorhanden (Abb. 3a). ${ }^{58}$ Beide Stücke waren stark fragmentiert. Die Ornamentik auf beiden Stücken ist nahezu identisch. Auf der Bodenunterseite sitzen kreisförmig angeordnet mehrere konzentrische Kreiskanneluren, in deren Mitte jeweils ein kleiner Buckel sitzt. Während die

55 Bei der Mehrzahl der im Detail analysierten Gefäße und Gefäßfragmente aus Monkodonja (3997 von 7420) konnte die Oberflächenbehandlung auf der Außenseite auf Grund der Erhaltungsbedingungen nicht mehr bestimmt werden.

56 Vgl. auch Hellmuth Kramberger 2017, 163, 404 und Abb. 281/b.

57 Hellmuth Kramberger 2017, Taf. 45/2.

58 Hellmuth Kramberger 2017, Taf. 46.
Anzahl der Kreise für die kleinere Schale auf Grund ihres Erhaltungszustandes nicht mehr eindeutig zu bestimmen ist ${ }^{59}$ sind es bei der großen Schale zweifelsfrei sieben konzentrische Kreise aus jeweils drei Kreiskanneluren - sechs Kreise, die um einen zentralen Kreis herum angeordnet sind. Das Motiv erinnert an die Verzierung auf runden Goldblechen, die im Schachtgrab III im Gräberrund A von Mykene gefunden wurden. ${ }^{60} \mathrm{Um}$ die sieben konzentrischen Kreise auf dem Boden der Schale aus Monkodonja sind kleine eingedrückte Vertiefungen, Punkte, verteilt. ${ }^{61}$ Ihre genaue Anzahl lässt sich nicht bestimmen, insgesamt 17 haben sich erhalten. Auf der Schalenaußenseite finden sich nun weitere Ziermotive, welche die gesamte untere Schalenhälfte einnehmen. Zunächst laufen drei konzentrische Kanneluren kreisförmig um den Boden herum, die von vier großen konzentrischen Kreiskanneluren, gebildet aus je vier Kreisen mit zentralem Buckel, geschnitten werden. Die konzentrischen Kreise mit zentralem Buckel sind antithetisch angeordnet bzw. weisen in jeweils eine Himmelsrichtung. Im Fall der größeren Schale sitzen jeweils unmittelbar auf der äußersten Kannelur, die um den Boden verläuft, sichelmondförmige Bögen, die aus jeweils zwei parallelen breiten Kanneluren gebildet sind. Im Fall der etwas kleineren Schale sind diese Bögen einige Zentimeter von der äußersten Kannelur um den Boden herum abgerückt. An den Enden der sichelmondförmigen Bögen sitzen kleine eingetiefte Punkte, identisch zu jenen auf der Bodenunterseite. Weitere Punkte sind in den „Zwickeln“ zwischen den Bögen und den konzentrischen Kreisen angebracht, wobei sich keine Regelhaftigkeit feststellen lässt. Während bei der kleineren Schale sowohl im linken, als auch im rechten Zwickel Punkte sitzen, finden sie sich auf der größeren Schale einmal einzeln im linken $Z$ wickel, als auch im rechten $Z_{\text {wi- }}$ ckel, wobei auf Grund des Erhaltungszustandes nicht mehr

59 Von drei Kreisen haben sich Teile erhalten (vgl. Abb. 2b): HeLLmUth Kramberger 2017, Taf. 45/2. Da sie in ihrer Rekonstruktion bereits etwas über die Hälfte des Bodendurchmessers einnehmen, ist es unwahrscheinlich, dass die gleiche Anzahl von Kreisen bestand, wie im Falle der größeren Schale. Auch ist fraglich, ob ein zentraler konzentrischer Kreis vorhanden war.

60 Karo 1930-1933, 44 und Taf. 28-29. - Marinatos, Hirmer 1973, Abb. 224/zweite Reihe. - Vgl. Hellmuth Kramberger 2017, 385-386 und Abb. 278-279.

61 Im Zentrum sitzen die Punkte jeweils im „Zwickel“ zwischen dem zentralen Kreis und zwei angrenzenden konzentrischen Kreisen, jeweils drei Punkte sitzen in dem „Zwickel“ zwischen Bodenrand und jeweils zwei Kreisen. Allerdings findet sich im Fall eines konzentrischen Kreises, dem vollständig erhaltenen auf der rechten Seite, noch ein zusätzlicher Punkt dicht gedrängt zwischen dem Bodenrand und dem konzentrischen Kreis. 


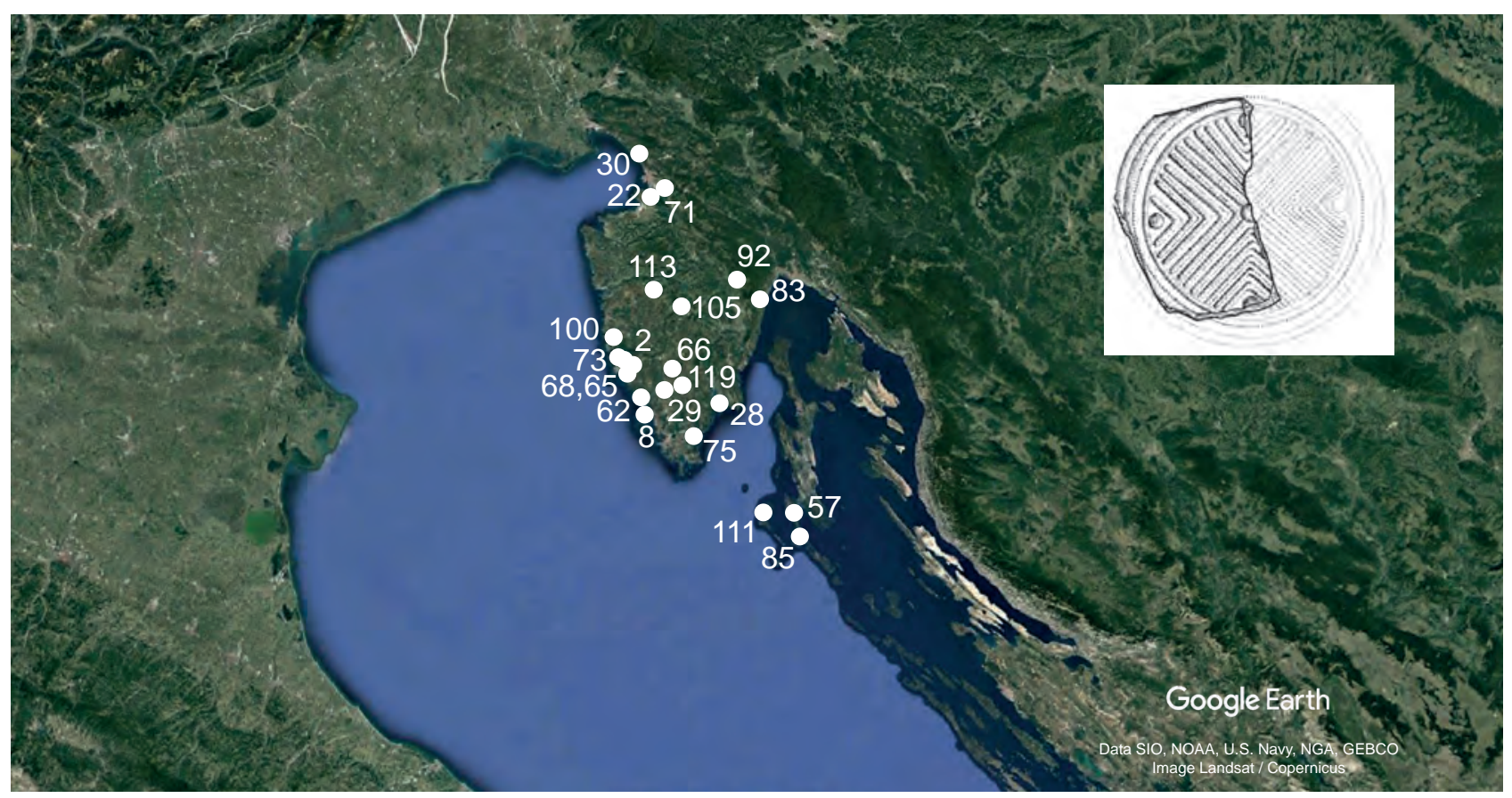

Abb. 5. Verbreitung von Schalen mit verzierter Unterseite in Istrien (nach Hellmuth Kramberger 2017, Abb. 134, ergänzt; Hintergrund: Google Earth). - 2. Bale/Valle (Buršić-Matijašić 2010, Taf. 4/74). - 8. Brijuni-Gradina (Cardarelli 1983, Taf. 24b/14, 16). - 22. Ėlleri/Jelarji (Maselli Scotтi 1997, Taf. 3/12-13). - 28. Gradac-Turan (Minovilić 1997, Taf. 1/3; 3/6-7; 4/11). - 29. Gropi-Stari Guran (Mihovilić 20072008, Taf. 3/24). - 30. Grotta Caterina (Canarella, Pitti 1978-1981, Abb. 4/3). - 57. Lošinj,Vela spilja, Insel (Mirosavljević 1968, Taf. 24/ oben links). - 62. Mandriol (Kaspar, KasPar 2005, 122, Foto rechts). - 65. Monbrodo (unpubliziert). - 66. Moncas di Valle/Monkas kod Bala di Valle (Cardarelli 1983, Taf. 24a/6). - 68. Monkodonja. - 71. Montedoro/Dolga Krona (Maselli Scotti 1997, Taf. 6/8). - 73. Monvi/ Monvè (Bekić 1996, Taf. 7/4). - 75. Nesactium/Nesazio (Minovilić 2001, Taf. 117/26). - 83. Podosojna peć/Podosojna Höhle (STARac 1991, Taf. 24/5). - 85. Polanža, Lošinj, Insel (CArdarelli 1983, Taf. 26/12). - 92. Pupićina peć/Pupićina Höhle (Forenbaher, Kaiser, Miracle 2003-2004, Abb. 9/5). - 100. Školjić-Funtana (Mihovilić 1995, Taf. 2/1). - 105. Sv. Marija Magdalena (Kaspar, Kaspar 2005, 53, Foto rechts unten). - 111. Unije, Gradina Turan, Insel (Miletić 2002, Taf. 4/9-10). - 113. Vela peć kod Vranje/Vela Höhle bei Vranje (Forenbaher, Rajić Šikanjić, Miracle 2006, Abb. 12/2). - 119. Vrčin/Monte Orcino (Buršić-Matijašić 1997, Taf. 9/129-139; 17/368).

zu bestimmen ist, ob es noch einen zweiten Punkt auf der linken Seite des entsprechenden Bogens gab. ${ }^{62}$

Auf das Vorkommen von Schalenverzierungen an der Bodenunterseite allgemein wurde bereits an anderer Stelle ausführlich eingegangen, sie stellen eines der charakteristischen Elemente der früh- und mittelbronzezeitlichen

62 Eine weitere Gruppe an Schalen mit verziertem Boden aus dem Bereich der Sonda VI bilden ebenfalls sehr große Exemplare, deren Ornamentik auf der Bodenunterseite in gewissem Sinne eine Kombination aus derjenigen an den vorab beschriebenen Stücken der identischen fünf kleinen und zwei großen Schalen darstellt (Abb. 1/B4). Der gesamte Boden wird von unterschiedlich breiten konzentrischen Kanneluren mit zentralem Buckel eingenommen, um diesen herum sind fünf konzentrische Kreise, ebenfalls mit zentralem Buckel, angebracht: Hellmuth Kramberger 2017, Taf. 43/4; 47; 48. - Weitere Fragmente von Schalen mit ähnlicher Motivik finden sich auch in Sonda IX (Hellmuth Kramberger 2017, Taf. 12/11), V (Taf. 70/4) und III (Taf. 107/6; 119/8).
Keramik in Istrien dar (Abb. 5). ${ }^{63}$ Es kann jedoch bemerkt werden, dass zwei Kontaktzonen zu benennen sind, die während des betreffenden Zeitraums ebenfalls Bodenverzierungen an Schalen kannten, wobei es teilweise sogar um identische Ziermotive geht: dies sind einerseits der norditalienische Raum mit dem Veneto und dem südlichen Gardaseegebiet, ${ }^{64}$ andererseits das Karpatenbecken mit Bodenverzierungen an Schalen im Mileu der Füzesabony-Gruppe bzw. -Kultur Ungarns ${ }^{65}$ sowie in Rumänien. ${ }^{66}$

63 Hellmuth Kramberger 2017, 161-168, 375-377.

64 Vgl. dazu auch: Urban 1993, 152-169, 160 und Abb. 80/b; 165 und Abb. 86; TeržAn, Mihovilić, HäNSEl 1999, 187.

65 Bóna 1975, Taf. 174/7.13.17; 175/18; 177/1.5; 178/11.13; 179/19; 180/1.5.10. - Hellmuth Kramberger 2017, 377.

66 Dumitrașcu, Emődi 1981. - Die Bodenverzierungen an Schalen der Wietenbergkultur Rumäniens zeigen hingegen kaum vergleichbare Ziermotive: vgl. BoroffkA 1994, 194 und Typentafel 18/21-26; 19-20/1-7. 
Genannt seien beispielsweise Schalen mit Bodenverzierung in Form von Buckeln, konzentrischen Kanneluren und Girlanden im Gräberfeld von Megyaszó (Abb. 1/B1). ${ }^{67}$ Dabei sei jedoch betont, dass sich für die beiden großen Schalen, die im Zentrum der Betrachtung stehen, keine Parallelen finden. Sie stellen in Monkodonja insgesamt etwas Besonderes dar und haben keine direkten Vergleiche, weder in Istrien, noch in den benachbarten oder weiter entfernten Regionen.

Schalen mit verziertem Boden kommen im gesamten gegrabenen Siedlungsbereich von Monkodonja vor, ${ }^{68}$ doch die größte Konzentration sowie die herausragenden bzw. besonderen Stücke finden sich ausschließlich in Sonda VI vor dem Eckbau bzw. Eckturm mit dem Steinkistengrab B. Welche Funktion erfüllten diese speziellen Schalen, waren sie womöglich Bestandteil von Zeremonien, die zu bestimmten Anlässen in der unmittelbaren Umgebung bzw. in der Anwesenheit der Ahnen in den Gräbern abgehalten wurden? Welche Rolle spielte die Ausrichtung des Tores gen Westen, in Richtung des Meeres und des Sonnenuntergangs zur Tagundnachtgleiche? ${ }^{29}$ Fragen, die sich heute freilich kaum beantworten lassen. Dennoch finden sich Hinweise darauf, dass es um besondere Objekte ging, deren Verzierung nicht nur eine ansprechende Ornamentierung repräsentierte, sondern einen speziellen Sinnträger darstellte. Bereits in einem anderen Zusammenhang hat sich gezeigt, dass bestimmte Verzierungen auf den Gefäßen in Monkodonja nicht nur schmückenden Charakter hatten, sondern aller Wahrscheinlichkeit nach auch Sinnträger waren. So konnte festgestellt werden, dass manche Töpfe und Pithoi, große Vorratsgefäße, auffällige Verzierungen tragen. ${ }^{70}$ Die kombinierten Motive aus bogenförmigen plastischen Leisten oder Einritzungen, kleinen Buckeln, Knöpfen und antithetisch angeordneten Handhaben formen "Gesichter“ und geben so den Gefäßen eine anthropomorphe Gestalt. Man mag sie als die „Hüter“ über den Inhalt der Gefäße interpretieren. Dass es sich nun auch bei den Verzierungen auf den beiden großen Schalen aus Monkodonja nicht um einen bloßen Schmuck, sondern vielmehr

67 Bóna 1975, Taf. 174/7.13.17; 175/18; 177/1.5; 178/11.13; 179/19; 180/1.5.10.13. - DAvid 2010, 476 und Abb. 41/4.6.

68 Hellmuth Kramberger 2017, 309-310 und Abb. 247.

69 Für das Nordtor von Monkodonja ist eine besondere Bedeutung relativ offensichtlich, denn so führt die zickzackförmig gestaltete Torgasse direkt zu einer $51 \mathrm{~m}$ tiefen Schachthöhle, deren Umfeld in prähistorischer Zeit überarbeitet und sehr wahrscheinlich kultisch genutzt worden war: HäNSEL, MiHovilić, TERŽAN 2015, 179-192, 381-387.

70 Hellmuth 2012. - Hellmuth 2015. um einen Sinnträger bzw. genauer, einen möglichen Bezug zu kosmologischen Vorstellungen handeln könnte, soll im Folgenden mit einem Vergleich diskutiert werden. Konzentrische Kreise, Buckel, eingetiefte Punkte und Bögen bilden, wie vorab beschrieben, die einzelnen Elemente. Erwähnt sei in diesem Zusammenhang, dass Wolfgang David das Vorkommen von Kreis- und Buckelverzierungen (flache Buckel und Spitzbuckel) auf der karpatenländischen Keramik der Stufen Bz A2-C nach mitteleuropäischer Terminologie, dabei speziell im Bereich der Füzesabony-Otomani- und Guylvarsánd-Otomani-Kultur, als Sinnträger angesprochen hat, der mit kosmologischen oder religiösen Vorstellungen in Verbindung zu bringen sei. ${ }^{71}$

\section{Astrologische Kenntnisse der Frühbronzezeit und eine mögliche Deutung der Ornamentik auf den beiden besonderen Schalen aus Monkodonja}

$\mathrm{Zu}$ den bemerkenswertesten Funden der mitteleuropäischen Frühbronzezeit gehört die sog. Himmelsscheibe aus dem Hortfund von Nebra, entdeckt auf dem Mittelberg im Burgenlandkreis Sachsen-Anhalts (Deutschland), die ohne Zweifel mit kosmologischen Vorstellungen in Zusammenhang steht (Abb. 6a-b). Viel wurde sowohl zur Geschichte der spektakulären Auffindung bzw. Sicherstellung des Hortes sowie der einzelnen Bestandteile als auch seiner Interpretation veröffentlicht. ${ }^{72}$ Deponiert wurde der Hortfund von Nebra, der dem kulturellen Milieu der mitteldeutschen Aunjetitz-Kultur zugeordnet wird, ${ }^{73}$ um 1600-1550 v. Chr., in der ausgehenden Frühbronzezeit (Bz A2 $\mathrm{c}^{74}$ bzw. Bz A3 $3^{75}$ nach der Phasengliederung für Zentraleuropa). Er kam damit zu einer Zeit in den Boden, als auch die befestigte Siedlung von Monkodonja in Istrien bestand. Abgesehen von der Himmelsscheibe ${ }^{76}$ umfasste der Hortfund auch zwei bronzene Vollgriffschwerter mit goldener Manschette am

\footnotetext{
71 DAvid 2010, 476-479. - Auch in Hinblick auf zeitgleiche Verzierungen auf Bronzen.

72 Aus dem umfangreichen Korpus an Veröffentlichungen sei an dieser Stelle nur eine Auswahl genannt: Melder 2002. - MelLer 2004. - Meller 2010. - Meller 2013a. - Meller 2013b. - Meller 2018. - Hansen 2010. - Pernicka et al. 2008. - Schlosser 2010. 73 Z. B. Meller 2002, 17. - Meller 2013a, 493. - Siehe Beitrag von E. Pernicka et al. in diesem Band, S. 89-122 [Anm. d. Red.]. 74 Meller 2013a, 500-503, 513, siehe auch Fußnote 26. 75 Meller 2002, 9.

76 Die Himmelsscheibe selbst war zu dem Zeitpunkt, als sie dem Boden übergeben wurde, entsprechend der Forschungsergebnisse bereits 150-200 Jahre alt: Meller 2010, 62. - Melder 2018, 356 und Abb. 6.
} 

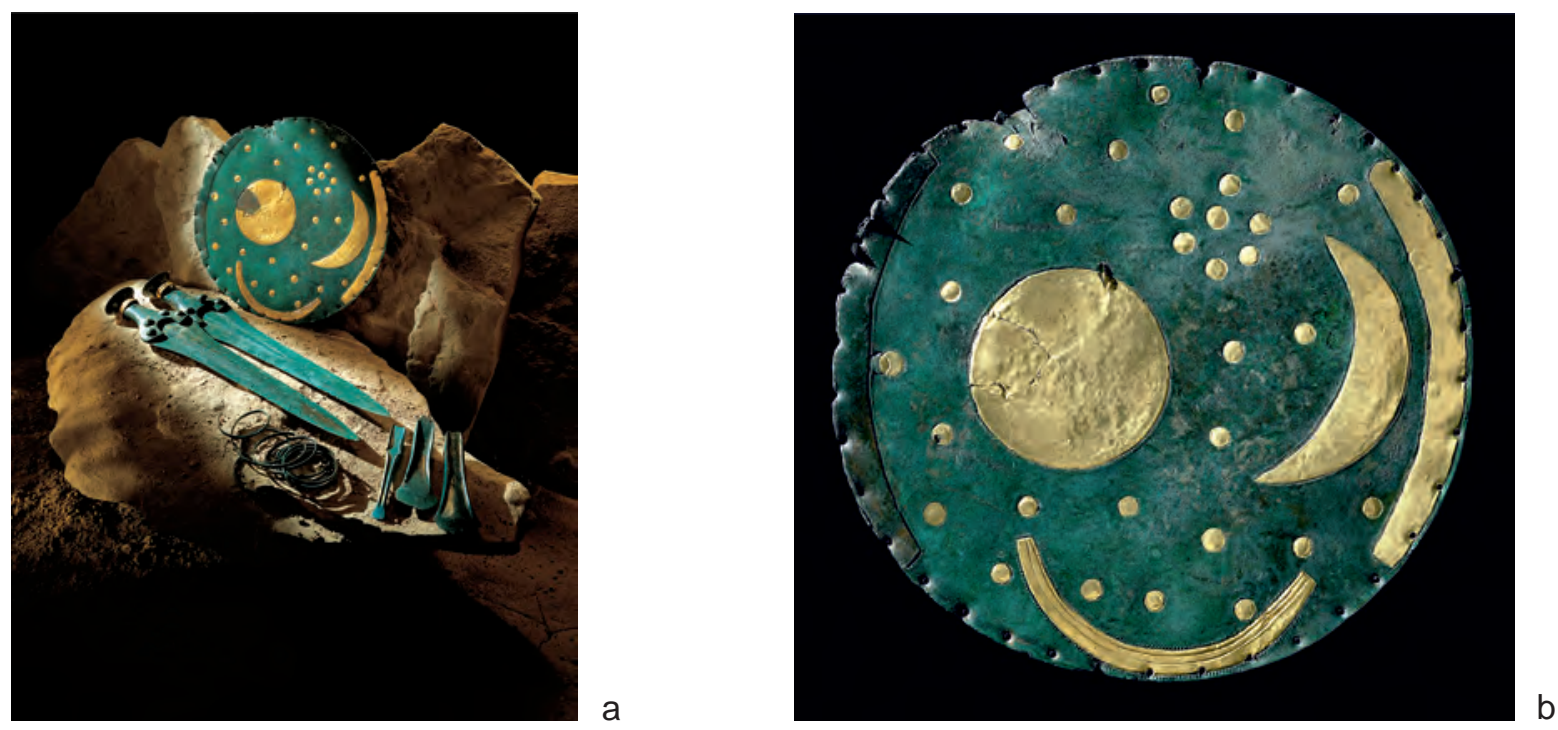

Abb. 6. - a. Der Hortfund von Nebra in rekonstruierter Fundlage (Foto: J. Lipták, @ Landesamt für Denkmalpflege und Archäologie Sachsen-Anhalt). - b. Die Himmelsscheibe von Nebra (Foto: J. Lipták, ( L Landesamt für Denkmalpflege und Archäologie Sachsen-Anhalt).

Griff, ${ }^{77}$ zwei Randleistenbeile, ${ }^{78}$ einen Knickrandmeißel ${ }^{79}$ sowie zwei mehrfach zerbrochene Armspiralen. ${ }^{80}$ Es wird angenommen, dass die Scheibe derart positioniert werden konnte, ${ }^{81}$ dass das rechte Ende des linken „Horizontbogens“ auf der Scheibe ${ }^{82}$ (vgl. Abb. 6b) den Sonnenuntergang zur Wintersonnenwende anzeigt, während das linke Ende desselben Bogens in Richtung des Sonnenuntergangs zur Sommersonnenwende weist. Letzteres konnte vom Mittelberg

77 Bei den beiden Schwertern handelt es sich aller Wahrscheinlichkeit nach um Kopien donauländischer Vollgriffschwerter, da sie einerseits Klingen besitzen, die jenen des Typs Sögel entsprechen, andererseits Griffe, die denen der Schwerter vom Typ Apa entsprechen: Meller 2010, 48-56. - Meller 2013a, 503-515.

78 Die Randleistenbeile werden dem Typ Bühl zugerechnet, der von K.-F. Rittershofer beschrieben wurde: MELLER 2010, 56. - Vgl. RiTTERSHOFER 1983, 178-189.

79 Der Meißel entspricht dem Typ 36a nach B. Zich: Meller 2010, 56. - Vgl. ZICH 1996, 214-215 und Karte 97.

80 Meller 2013a, 496-498 und Abb. 2/a-b; 3; 5-6. - Niedergelegt war der Hort, den Erkenntnissen der Ausgrabungen im Bereich des „Raublochs“ auf der Kuppe des Mittelberges zufolge, an einer markanten natürlichen Steinschichtung, wobei die Himmelsscheibe senkrecht stehend an einen Stein angelehnt gewesen sein soll, die übrigen Gegenstände unmittelbar vor ihr übereinander aufgeschichtet. Die Schwerter lagen dabei zuunterst übereinander, darüber im Wechsel ein Beil, der Knickrandmeißel und wiederum ein Beil, die Armspiralen befanden sich auf der rechten Seite.

81 Bzw. positioniert werden musste, da die Beobachtungen nur gemacht werden konnten, wenn die Scheibe dem Sternenhimmel gleich über den Kopf gehalten wird: MelLer 2018, 357.

82 Von der "Sonnenbarke“ in der Mitte am Rand der Scheibe aus betrachtet der linke Bogen. aus während der Frühbronzezeit auf Höhe des Brockens im Harzmassiv beobachtet werden. ${ }^{83}$ Umgekehrt zeigten die Enden des rechten „Horizontbogens“ auf die Sonnenaufgänge. Offensichtlich war die Nutzung der Scheibe auf die spezifischen Begebenheiten ihres Auffindungsortes zugeschnitten, wobei technische und naturwissenschaftliche Analysen ergeben haben, dass das Bildwerk auf der Scheibe in vier Phasen entstanden sei, also mehrfach modifiziert bzw. angepasst wurde, wobei drei unterschiedliche Goldsorten Verwendung fanden. ${ }^{84}$

Dabei umfasste das Bildwerk in seiner ersten Phase zunächst 32 Sterne, die Mondsichel ${ }^{85}$ sowie den Vollmond/ die Sonne, welches in der zweiten Phase durch die beiden „Horizontbögen“ erweitert wurde. In der dritten Phase wurde die „Sonnenbarke“ in der Mitte der beiden Bögen hinzugefügt. Die vierte Phase umfasst die Perforierung des Randes der Scheibe, sehr wahrscheinlich, um sie auf einem organischen Untergrund zu fixieren. In einer letzten, fünften Phase wurde der linke „Horizontbogen“ wieder entfernt, was womöglich geschah, um die Scheibe vor ihrer Deponierung unbrauchbar zu machen bzw. zu entwerten. Die Anpassung auf die astronomischen Gegebenheiten am Mittelberg erfolgte demnach mit der zweiten Phase, wobei entsprechend der Goldanalysen nicht nur die Bögen (bzw.

83 Schlosser 2004. - Schlosser 2010. - Meller 2018, 357. 84 Meller 2010, 46-48 und Abb. 15-16; 59-70 und Abb. 35. 85 In Frage kommt auch eine Deutung als partielle Sonnen- oder Mondfinsternisse: SCHLOSSER 2004, 46. 
der eine erhaltene Bogen) angebracht wurden, sondern auch ein einzelner Stern (Nr. 23) ${ }^{86}$ Insgesamt wird vermutet, dass in der Vorstellungswelt der Frühbronzezeit der Himmel als kuppelförmiges Gebilde angesehen wurde und die Himmelsscheibe von Nebra somit „die früheste Abstraktion dieses dreidimensionalen Gedankens in eine zweidimensionale Form“ verkörpert. ${ }^{87}$ Der gefiederte und als Schiff („Sonnenbarke“) interpretierte Bogen zwischen den beiden „Horizontbögen “ könnte dabei eine mythologischreligiöse Komponente verkörpern, deren genaue Deutung sich jedoch unserer Kenntnisse entzieht. ${ }^{88}$ Eine besondere Bedeutung kommt aus astronomischer Sicht einer Gruppe aus sieben Sternen zu, die sich rechts oberhalb der Sichel findet (Abb. 6b). Diese Gruppe stellt mit hoher Wahrscheinlichkeit den offenen Sternenhaufen der Plejaden dar, das „Siebengestirn“, der sich am Himmel mit bloßem Auge erkennen lässt und der vermutlich das zentrale Objekt auf der Himmelsscheibe bildet. ${ }^{89}$ Dem Siebengestirn bzw. auch der Zahl Sieben an sich kam in der mythologischen und religiösen Vorstellungswelt Mesopotamiens eine besondere Bedeutung zu. ${ }^{90}$ In der babylonischen Astrologie wurden die böse Sieben mit den Plejaden identifiziert, die auch durch eine siebenköpfige Schlange versinnbildlicht waren, der eine Gruppe der guten Sieben gegenüberstand. ${ }^{91}$ Ein zentraler Bestandteil der babylonischen Astrologie war die Beobachtung und Dokumentation des ersten Erscheinens und Verschwindens von Sternen und Planeten in der Morgen- und Abenddämmerung nahe des Horizontes. Eine Sammlung von Keilschrifttexten des 3.-1. Jts. v. Chr., die derartige Informationen enthält, sind die MUL.APINTafeln, in denen die Plejaden als MUL.MUL benannt sind. ${ }^{92}$ Auf dem Mittelberg in Sachsen-Anhalt waren die Plejaden in der Frühbronzezeit in der Dämmerungshelligkeit innerhalb von zwei bis drei Tagen unsichtbar und zwar einerseits um den 10. März, andererseits um den 17. Oktober, was man in etwa mit dem Beginn und dem Ende des bäuerlichen Jahres gleichsetzen kann. ${ }^{93}$ Wie Wolfhard Schlosser bemerkt, ${ }^{94}$ wurden Plejadenbeobachtungen beispielsweise in Litauen noch bis in die jüngste Vergangenheit genutzt,

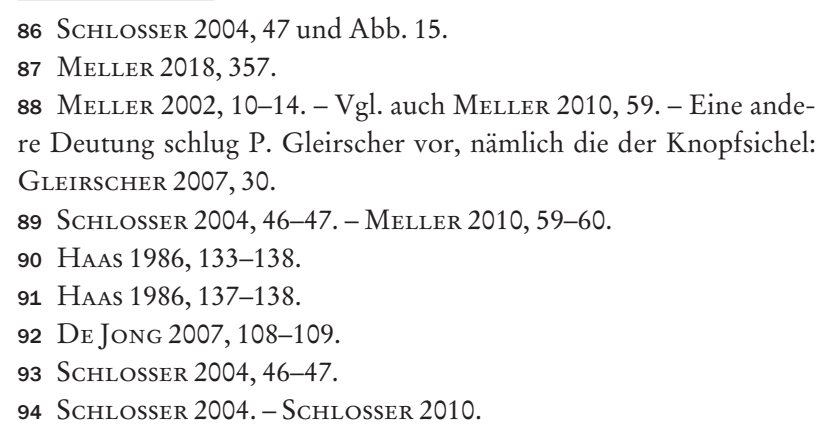

um den rechten Zeitpunkt zum Vorbereiten der Frühjahrssaat zu bestimmen, und eine ähnliche Nutzung erscheint unter Anbetracht der aufgeführten Begebenheiten auch für die Himmelsscheibe von Nebra wahrscheinlich.

Die Ikonografie der Himmelsscheibe von Nebra ist in dieser Art bislang für die entwickelte Frühbronzezeit Mitteleuropas einzigartig. Wenn nun auch in ihren jeweiligen Details unterschiedlich und auf unterschiedlichen Objekten und Materialien angebracht, so lassen sich dennoch gewisse Parallelen zwischen der Ornamentik bzw. einzelnen Elementen auf den beiden zuvor besprochenen Schalen aus Monkodonja und der Himmelsscheibe von Nebra feststellen. Die offensichtlichste Parallele stellen dabei die sichelmondförmigen Bögen dar. Der doppelt geriefte und „gefiederte“, d. h. mit feinen senkrechten Linien umsäumte Bogen auf der Himmelsscheibe von Nebra wird in der Regel als Schiff, als „Sonnenbarke“ interpretiert. ${ }^{95}$ Auf den Schalen aus Monkodonja finden sich jeweils vier (in jeder Himmelsrichtung eine) solche „Barken“, die ebenfalls gerieft bzw. in diesem Fall kanneliert, nicht aber gefiedert sind. Die „Sonnenbarke" auf der Himmelsscheibe sei, wie weiter oben bemerkt, erst in der dritten Fertigungsphase hinzugefügt worden. ${ }^{96}$ Dennoch ist interessant, dass sie derart eingefügt wurde, dass sich, wenn auch leicht versetzt, jeweils oberhalb der Enden des Bogens ein Stern befindet; ein einzelner Stern findet sich auch in dem Zwickel zwischen dem Scheibenrand und der linken Hälfte des Bogens (Abb. 6b). Punkte (Sterne?) oberhalb der Bogenenden finden sich auch bei den Schalen aus Monkodonja, und in einem Fall wurde ebenfalls ein einzelner Punkt in dem Zwickel zwischen der linken Bogenhälfte und der äußersten Kannelur angebracht, die um den Boden herumläuft. Im Fall der größeren Schale sitzen die Bögen, die „Barken“, wie bemerkt, unmittelbar auf dieser äußersten Kannelur auf und es erscheint reizvoll, letztere als Horizont zu interpretieren. Aus diesem Horizont tauchen dann die großen konzentrischen Kreise mit zentralem Buckel („Tutulus“) auf bzw. in diesen ab. Sie könnten demnach entweder den Vollmond oder die Sonne darstellen, der bzw. die sich auch als zentraler Bestandteil bei der NebraScheibe findet. Und auch ein weiteres zentrales Motiv der Himmelsscheibe von Nebra findet sich möglicherweise auf der größeren Schale aus Monkodonja - das Siebengestirn, die Plejaden. Repräsentiert könnten diese in Form der sieben konzentrischen Kreise auf der Bodenunterseite sein, die von zahlreichen Punkten, Sternen, umgeben sind.

Eine Frage, die sich bei dem vorliegenden Vergleich stellt, ist freilich, ob eine ähnliche kosmologische Ikonografie und

95 Meller 2002, 10-14. - Vgl. auch Meller 2010, 59.

96 Meller 2010, 46-48 mit Abb. 16; 65-67. 
wahrscheinlich auch damit verknüpfte Vorstellungswelt in den beiden geografisch weit entfernten Regionen bestanden haben könnte, ohne dabei jedoch so weit gehen zu wollen, dem Töpfer in Istrien Kenntnisse von der Himmelsscheibe zu unterstellen. Wie eingangs ausgeführt wurde, zeigen die Funde aus der Gradina Monkodonja deutlich, dass Istrien in das weitreichende früh- und mittelbronzezeitliche Kommunikationsnetz Mitteleuropas eingebunden war und sich insbesondere Verbindungen in das mittlere Donaugebiet mit jenen Gruppen, die einen mehr oder minder starken Bezug zur böhmischen Aunjetitz-Kultur aufweisen, das Karpatenbecken sowie nach Norditalien ${ }^{97}$ nachverfolgen lassen. Einen Beleg dafür bilden die Brotlaibidole, bestimmte Bronzen und ferner auch ein zum Teil ähnliches Gefäßspektrum mit gemeinsamen Charakteristika sowie mutmaßliche Importe. Auf der anderen Seite stellt Istrien bzw. der Raum um Monkodonja den nördlichsten Ausläufer des Mittelmeeres dar und dürfte somit die Funktion eines Umschlagplatzes für Waren, Personen und Ideen zwischen dem Süden und Kontinentaleuropa gehabt haben. ${ }^{98}$ Vor diesem Hintergrund erscheint es also möglich, dass es sich bei den aufgezeigten Parallelen zwischen den Verzierungen auf den beiden Schalen aus Monkodonja und der Ikonografie der Himmelsscheibe von Nebra nicht um einen bloßen „Zufall“ handelt, sondern sich auch in Istrien ähnliche astrologische Kenntnisse und vielleicht damit verknüpfte spirituelle Vorstellungen fassen lassen.

\section{Danksagung}

Dieser Artikel wurde durch die slowenische Forschungsförderungsorganisation (ARRS) im Rahmen der Forschergruppe „Raziskave kulturnih formacij / Research of Cultural Formations (P6-0278 (A), 2019-2024)“ an der Alma Mater Europaea - Institutum Studiorum Humanitatis, Fakulteta za podiplomski humanistični študij Ljubljana unterstützt.

\section{Literatur}

BARTELHEIM 1998

M. Bartelheim, Studien zur böhmischen Aunjetitzer Kultur Chronologische und chorologische Untersuchungen. Universitätsforschungen zur prähistorischen Archäologie 46, Bonn 1998.

BEKIĆ 1996

L. BekIć, Sustav gradina na Rovinjskom području, Histria Archaeologica 27, 1996, 19-92.

\section{Siehe auch Hellmuth Kramberger 2017, 346-353.}

98 Hänsel, Mihovilić, Teržan 2015, 501. - Zu den Südkontakten Monkodonjas: Hänsel, Minovilić, TeRŽAn 2015, 175-177, 500504. - Hellmuth Kramberger 2017, 379-386. - Hänsel, Teržan 2020, 193-195 und Abb. 17. - Ein Handelsgut, das offensichtlich vom nördlichen Mitteleuropa über Istrien weiter nach Süden gehandelt wurde, stellt der Bernstein dar, der zahlreich in den Grabhügeln auf dem Nachbarhügel Mušego gefunden wurde: HäNSEL et al. 2011. HäNSEl, Mihovilić, TerŽAn 2015, 501.

\section{BERTEMES 1989}

F. BERTEMES, Das frühbronzezeitliche Gräberfeld von Gemeinlebarn: Kulturhistorische und paläometallurgische Studien. Saarbrücker Beiträge zur Altertumskunde 45, Bonn 1989.

Bóna 1975

I. BónA, Die Mittlere Bronzezeit Ungarns und ihre südöstlichen Beziehungen. Budapest 1975.

BOROFFKA 1994

N. BoroffKA, Die Wietenberg-Kultur: Ein Beitrag zur Erforschung der Bronzezeit in Südosteuropa. Universitätsforschungen zur prähistorischen Archäologie 19, Bonn 1994.

BuRŠIĆ-MATIJAŠIĆ 1997

K. Buršić-Matijašić, Ceramica del Castelliere di Monte Orcino in Istria / Keramika gradine Vrčin u Istri, Histria Archeologica 28, 1997, 108-134.

BuRŠIĆ-MATIJAŠIĆ 1998

K. BuRšıć-Matijašıć, Gradina Monkodonja: Tipološko-statistička obrada keramičkih nalaza srednjobrončanodobne istarske gradine Monkodonja kod Rovinja / The Monkodonja Hillfort: A Typological and Statistical Analysis of Pottery Finds from the Middle Bronze Age Hillfort of Monkodonja near Rovinj. Monografije i katalozi Arheološki muzej Istre 9, Pula 1998.

BuRŠIĆ-MATIJAŠIĆ 2010

K. BuRŠić-MatiJašIć, Bale i keramički materijal iz palače Soardo-Bembo (Istraživanje 2000-2003) / Bale and the pottery from the Soardo-Bembo castle (research 2000-2003), Opuscula Archaeologica 34, 2010, 7-35.

Canarella, Pitti 1978-1981

D. Canarella, C. Pitti, Gli scavi nella Caverna Caterina sul carso Triestino, Atti della Società per la Preistoria e Protostoria della Regione Friuli-Venezia Giulia IV, 1978-1981, 9-32.

Cardarelli 1983

A. Cardarelli, Castellieri nel Carso e nell'Istria: Cronologia degli Insediamenti fra Media Età del Bronzo e Prima Età del Ferro. In: Preistoria del Caput Adriae (Ausstellungskatalog, Triest, Castello di S. Giusto 1983). Udine 1983, 87-112.

DAvid 2010

W. DAvid, Die Zeichen auf der Scheibe von Nebra und das altbronzezeitliche Symbolgut des Mitteldonau-Karpatenraumes. In: H. Meller, F. Bertemes (Hrsg.), Der Griff nach den Sternen: Wie Europas Eliten zu Macht und Reichtum kamen. Internationales Symposium in Halle (Saale), 16.-21. Februar 2005. Tagungen des Landesmuseums für Vorgeschichte Halle 5, Halle (Saale) 2010, 439-486.

De Jong 2007

T. DE Jong, Astronomical dating of the rising star list in MUL.APIN. In: Festschrift für Hermann Hunger zum 65. Geburtstag gewidmet von seinen Freunden, Kollegen und Schülern. Wiener Zeitschrift für die Kunde des Morgenlandes 97, Wien 2007, 107-120.

Dumitrașcu, Emődi 1981

S. Dumitrașcu, I. Emó́Di, Descoperiri arheologice hallstattiană de la Biharea, Ziridara XIII, 1981, 75-109.

Forenbaher, Kaiser, Miracle 2003-2004

S. Forenbaher, T. Kaiser, P. T. Miracle, Pupićina cave pottery and the neolithic sequence in northeastern Adriatic, Atti della Società per la Preistoria e Protostoria della Regione Friuli-Venezia Giulia XIV, 2003-2004, 61-102.

Forenbaher, Rajić ŠIKanjić, Miracle 2006

S. Forenbaher, P. Rajić Šikanjić, P. T. Miracle, Lončarija iz Vele Peći kod Vranje (Istra), Histria Archeologica 37, 2006, 5-46. 
GLeirscher 2007

P. Gleirscher, Zum Bildprogramm der Himmelsscheibe von Nebra: Schiff oder Sichel? Germania 85, 2007, 23-33.

HAAS 1986

V. HaAs, Magie und Mythen in Babylonien: Von Dämonen, Hexen und Beschwörungspriestern. Gifkendorf 1986.

HÄNSEL 2002

B. HäNSEL, Stationen der Bronzezeit zwischen Griechenland und Mitteleuropa, Bericht der Römisch-Germanischen Kommission 83, 2002, 69-97.

HÄNSEL, TERŽAN 2000

B. Hänsel, B. TeržAn, Ein bronzezeitliches Kuppelgrab außerhalb der mykenischen Welt im Norden der Adria, Prähistorische Zeitschrift 75, 2000, 161-183.

HäNSEL, TERŽAN 2020

B. Hänsel, B. Teržan, Metalni predmeti i drugi pojedinačni nalazi od kamena, gline, kosti i veprovog zuba / Metallgegenstände und andere Funde aus Stein, Ton, Knochen und Eberzahn. In: B. Hänsel, K. Mihovilić, B. TeržAn, Monkodonja: Istraživanje protourbanog naselja brončanog doba Istre 3: Nalazi od metala, gline, kosti i kamena kao i ljudskih i životinjskih kostiju / Monkodonja: Forschungen zu einer protourbanen Siedlung der Bronzezeit Istriens 3: Die Funde aus Metall, Ton, Knochen und Stein sowie die menschlichen und tierischen Knochen. Monografije i katalozi Arheološki muzej Istre 34, Pula 2020, 147-250.

Hänsel, Mihovilić, TERžAn 2015

B. Hänsel, K. Mihovilić, B. Teržan, Monkodonja: Istraživanje protourbanog naselja brončanog doba Istre 1: Iskopavanje i nalazi građevina / Monkodonja: Forschungen zu einer protourbanen Siedlung der Bronzezeit Istriens 1: Die Grabung und der Baubefund. Monografije i katalozi Arheološki muzej Istre 25, Pula 2015.

Hänsel, Mihovilić, TERŽan 2020

B. Hänsel, K. Mihovilić, B. TeržAn, Monkodonja: Istraživanje protourbanog naselja brončanog doba Istre 3: Nalazi od metala, gline, kosti i kamena kao i ljudskih i životinjskih kostiju / Monkodonja: Forschungen zu einer protourbanen Siedlung der Bronzezeit Istriens 3: Die Funde aus Metall, Ton, Knochen und Stein sowie die menschlichen und tierischen Knochen. Monografije i katalozi Arheološki muzej Istre 34, Pula 2020.

HäNSEL et al. 2009

B. Hänsel, D. MatošEvić, K. Mihovilić, B. Teržan, Zur Sozialarchäologie der befestigten Siedlung von Monkodonja (Istrien) und ihrer Gräber am Tor, Prähistorische Zeitschrift 84, 2009, 151-180.

HäNSEL et al. 2011

B. Hänsel, K. Mihovilić, D. Matošević, B. Teržan, Burial mounds of the Bronze Age at Mušego near Monkodonja: results of the excavations 2006-2007. In: E. Borgna, S. Müller CelkA (Hrsg.), Ancestral Landscapes: Burial Mounds in the Copper and Bronze Ages (Central and Eastern Europe - Balkans - Adriatic - Aegean, $4^{\text {th }}-2^{\text {nd }}$ millennium B.C.). Proceedings of the International Conference held in Udine, May $15^{\text {th }}-18^{\text {th }} 2008$. Travaux de la Maison de l'Orient de la Méditerranée, Lyon 2011, 367-374.

HANSEN 2010

S. Hansen, Der Hort von Nebra: Seine Ausstattung. In: H. Meller, F. Bertemes (Hrsg.), Der Griff nach den Sternen: Wie Europas Eliten zu Macht und Reichtum kamen. Internationales Symposium in Halle (Saale), 16.-21. Februar 2005. Tagungen des Landesmuseums für Vorgeschichte Halle 5, Halle (Saale) 2010, 77-89.
Hellmuth 2012

A. Hellmuth, Čuvari hrane i pica: O antropomorfnim ukrasima na posudama iz ranog i srednjeg brončanog doba s gradine Monkodonja u Istri / Guardians of food and drink: about anthropomorphic vessel decorations of the Early and Middle Bronze Age from Monkodonja hillfort in Istria, Histria Archaeologica 43, 2012, 19-46.

Hellmuth 2014

A. Hellmuth, Butter, Wein oder Honig? Betrachtungen zu einer besonderen Gefäßbodenform aus der Gradina von Monkodonja bei Rovinj in Istrien. In: S. Tecco Hvala (Hrsg.), Studia Praehistorica in Honorem Janez Dular. Opera Instituti Archaeologici Sloveniae 30, Ljubljana 2014, 65-79.

Hellmuth 2015

A. Hellmuth, Apotropaic elements on ceramics from the Early and Middle Bronze Age in the northern Adriatic region (Caput Adriae), Mediterranean Review 8/1, 2015, 1-28.

Hellmuth Kramberger 2017

A. Hellmuth Kramberger, Monkodonja: Istraživanje protourbanog naselja brončanog doba Istre 2: Keramika / Monkodonja: Forschungen zu einer protourbanen Siedlung der Bronzezeit Istriens 2: Die Keramik. Monografije i katalozi Arheološki muzej Istre 28, Pula 2017.

Hinz 2009

M. Hinz, Eine multivariate Analyse Aunjetitzer Fundgesellschaften. Universitätsforschungen zur prähistorischen Archäologie 173, Bonn 2009.

KALICZ 1968

N. KaliCz, Die Frühbronzezeit in Nordost-Ungarn: Abriss der Geschichte des 19.-16. Jahrhunderts v. u. Z. Archaeologia Hungarica 45, Budapest 1968.

KARO 1930-1933

G. Karo, Die Schachtgräber von Mykenai. München 1930-1933.

KASPAR, KASPAR 2005

H.-D. Kaspar, E. Kaspar, Istrien: Eine archäologische Entdeckungsreise. Schonungen 2005.

LAUERMANN 2003

E. Lauermann, Studien zur Aunjetitz-Kultur im nördlichen Niederösterreich. Universitätsforschungen zur prähistorischen Archäologie 99, Bonn 2003.

LOCHNER 2012

M. Lochner, Thunau am Kamp - Eine befestigte Höhensiedlung der Urnenfelderkultur und der außergewöhnliche Fund eines Tonfässchens. In: W. BlajER (Hrsg.), Peregrinationes archaeologicae in Asia et Europa Joanni Chochorowski dedicatae. Krakau 2012, 193-203.

Marinatos, Hirmer 1973

S. Marinatos, M. Hirmer, Kreta, Thera und das mykenische Hellas. München 1973.

Maselli SCOTTI 1997

F. Maselli Scot'ti (Hrsg.), Il Civico Museo Archeologico di Muggia. Triest 1997.

MAYER 1977

E. F. Mayer, Die Äxte und Beile in Österreich. Prähistorische Bronzefunde 9, München 1977.

Meller 2002

H. Meller, Die Himmelsscheibe von Nebra: Ein frühbronzezeitlicher Fund von außergewöhnlicher Bedeutung, Archäologie in Sachsen-Anhalt N.F. 1, 2002, 7-20. 
Meller 2004

H. Meller (Hrsg.), Der geschmiedete Himmel: Die weite Welt im Herzen Europas vor 3600 Jahren (Begleitband zur Sonderausstellung, Landesmuseum für Vorgeschichte Halle (Saale) 15.10.2004-24.4.2005, Dänisches Nationalmuseum Kopenhagen 1.7.2005-22.10.2005, Reiss-Engelhorn-Museen Mannheim 4.3.2006-9.7.2006). Stuttgart 2004.

Meller 2010

H. Meller, Nebra: Vom Logos zum Mythos - Biographie eines Himmelsbildes. In: H. Meller, F. Bertemes (Hrsg.), Der Griff nach den Sternen: Wie Europas Eliten zu Macht und Reichtum kamen. Internationales Symposium in Halle (Saale), 16.-21. Februar 2005. Tagungen des Landesmuseums für Vorgeschichte Halle 5, Halle (Saale) 2010, 23-73.

Meller 2013a

H. Meller, Der Hortfund von Nebraim Spiegel frühbronzezeitlicher Deponierungssitten. In: H. Meller, F. Bertemes, H.-R. Bork, R. Risch (Hrsg.), 1600 - Kultureller Umbruch im Schatten des Thera-Ausbruchs? 4. Mitteldeutscher Archäologentag vom 14. bis 16. Oktober 2011 in Halle. Tagungen des Landesmuseums für Vorgeschichte Halle 9, Halle (Saale) 2013, 493-526.

MELler 2013b

H. Meller, The sky disc of Nebra. In: H. Fokkens, A. Harding (Hrsg.), The Oxford Handbook of the European Bronze Age. Oxford 2013, 266-269.

MELLER 2018

H. Meller, Das Wissen um Zeit und Raum: Himmelsdarstellungen in der Bronzezeit. In: M. Wemhoff, M. M. Rind (Hrsg.), Bewegte Zeiten: Archäologie in Deutschland. Petersberg 2018, 351-359.

MiHovilić 1995

K. Mihovilıć, Školjić (Funtana) i tragovi prapovijesnih obalnih i otočnih lokaliteta Istre, Histria Archeologica 26, 1995, 28-57.

Mihovilić 1997

K. Minovilıć, Fortifikacija gradine Gradac-Turan iznad Koromačna / The fortification of Gradac or Turan hill-fort above Koromačno, Izdanja Hrvatsko arheološko društvo 18, 1997, 39-59.

Mihovilić 2001

K. Minovilıć, Nezakcij: Prapovijesni nalazi 1900-1953 / Nesactium: Prehistoric Finds 1900-1953. Monografije i katalozi Arheološki muzej Istre 11, Pula 2001.

Mihovilić 2007-2008

K. Minovilıć, Gropi - Stari Guran: analiza prapovijesne keramike, Histria Archeologica 38-39, 2007-2008, 37-79.

Minovilić 2020

K. MiHovilić, Krušni idoli - enigmatske pločice - pločice s otiscima iz Monkodonje / Brotlaibidole - enigmatische Täfelchen - Täfelchen mit Stempeleindrücken aus Monkodonja. In: B. HäNSEL, K. Mihovilić, B. TeržAn, Monkodonja: Istraživanje protourbanog naselja brončanog doba Istre 3: Nalazi od metala, gline, kosti i kamena kao i ljudskih i životinjskih kostiju / Monkodonja: Forschungen zu einer protourbanen Siedlung der Bronzezeit Istriens 3: Die Funde aus Metall, Ton, Knochen und Stein sowie die menschlichen und tierischen Knochen. Monografije i katalozi Arheološki muzej Istre 34, Pula 2020, 115-146.

Mihovilić, Hänsel, Teržan 2011

K. Mihovilić, B. Hänsel, B. Teržan, Monkodonja: Rovigno (Croazia). In: A. Piccoli, R. Laffranchini (Hrsg.), Enigma: Un Antico Processo di Interazione Europea: Le Tavolette Enigmatiche / Enigma: An Ancient European Interaction: The Enigmatic Tablets. Cavriana 2011, 131-136.
Mihovilić, Hänsel, TERŽAn 2017

K. Mihovilić, B. Hänsel, B. TeržAn, Brončanodobni krušni idoli: enigmatiske pločice ili...? / Talismani dell'Età del Bronzo: Tavolette Enigmatiche, o...? / Bronze Age Loaf-of-Bread Idols: Enigmatic Tablets...or? Katalogi izložbi 5, Pula 2017.

Miletić 2002

I. Miletić, Arheološka topografija otoka Unije, Histria Archeologica 33, 2002, 195-263.

Mirosavljević 1968

V. Mirosavljević, Vela spilja prethistorijsko nalažište na otoku Lošinju, Arheološki radovi i rasprave VI, 1968, 27-52.

Moucha 1963

V. Moucha, Die Periodisierung der Úněticer Kultur in Böhmen, Sborník Československé společnosti archeologické 3, 1963, 9-60.

Neugebauer 1976

J.-W. Neugebauer, 25 Jahre Bronzezeitforschung in Niederösterreich: Eine Zusammenfassung des Forschungsstandes auf dem Gebiete der frühen und mittleren Bronzezeit, Archaeologia Austriaca 59-60, 1976, 49-86.

Neugebauer 1977

J.-W. Neugebauer, Böheimkirchen: Monographie des namengebenden Fundortes der Böheimkirchnergruppe der Věteřovkultur. Die Grabungen der Jahre 1929, 1945-1949 und 1955, Archaeologia Austriaca 61/62, 1977, 31-207.

Neugebauer 1987

J.-W. Neugebauer, Die Bronzezeit im Osten Österreichs. Forschungsberichte zur Ur- und Frühgeschichte 13, St. Pölten Wien 1987.

Neugebauer 1994

J.-W. Neugebauer, Bronzezeit in Ostösterreich. St. Pölten - Wien 1994.

Neugebauer, Blesl 1998

J.-W. Neugebauer, C. Blest, Das Traisental in Niederösterreich: Die Siedlungserschließung einer Tallandschaft im Alpenvorland in der Bronzezeit. In: B. HäNSEL (Hrsg.), Mensch und Umwelt in der Bronzezeit. Kiel 1998, 395-418.

Neugebauer, Neugebauer 1997

C. Neugebauer, J.-W. Neugebauer, Franzhausen: Das frühbronzezeitliche Gräberfeld I. Fundberichte aus Österreich, Materialhefte A5/2, Wien 1997.

PERnicka et al. 2008

E. Pernicka, C.-H. Wunderlich, A. Reichenberger, H. Meller, G. Borgh, Zur Echtheit der Himmelsscheibe von Nebra: Eine kurze Zusammenfassung der durchgeführten Untersuchungen, Archäologisches Korrespondenzblatt 38, 2008, 331-352.

Piccoli, Laffranchini 2011

A. Piccoli, R. Laffranchini (Hrsg.), Enigma: Un Antico Processo di Interazione Europea: Le Tavolette Enigmatiche / Enigma: An Ancient European Interaction: The Enigmatic Tablets. Cavriana 2011.

RITTERSHOFER 1983

K.-F. RitTershofer, Der Hortfund von Bühl und seine Beziehungen, Bericht der Römisch-Germanischen Kommission 64, 1983, 139-415.

SCHAMBERgER 2007

E. Schamberger, Die bronzezeitlichen Siedlungsreste aus Vorwald bei Wald am Schoberpass, Stmk. In: G. Tiefengraber (Hrsg.), Studien zur Mittel- und Spätbronzezeit am Rande der Südostalpen. Universitätsforschungen zur prähistorischen Archäologie 148, Bonn 2007, 235-327. 
Schlosser 2004

W.Schlosser, Die Himmelsscheibe von Nebra: Astronomische Untersuchungen. In: H. Meller (Hrsg.), Der geschmiedete Himmel: Die weite Welt im Herzen Europas vor 3600 Jahren (Begleitband zur Sonderausstellung, Landesmuseum für Vorgeschichte Halle (Saale) 15.10.2004-24.4.2005, Dänisches Nationalmuseum Kopenhagen 1.7.2005-22.10.2005, Reiss-Engelhorn-Museen Mannheim 4.3.2006-9.7.2006). Stuttgart 2004, 44-47.

SCHLOSSER 2010

W. Schlosser, Die Himmelsscheibe von Nebra: Astronomische Untersuchungen. In: H. Meller, F. Bertemes (Hrsg.), Der Griff nach den Sternen: Wie Europas Eliten zu Macht und Reichtum kamen. Internationales Symposium in Halle (Saale), 16.-21. Februar 2005. Tagungen des Landesmuseums für Vorgeschichte Halle 5, Halle (Saale) 2010, 913-933.

SCHNEIDER 1989

G. SchneIder, Naturwissenschaftliche Kriterien und Verfahren zur Beschreibung von Keramik: Diskussionsergebnisse der Projektgruppe Keramik im Arbeitskreis Archäometrie in der Fachgruppe Analytische Chemie der Gesellschaft Deutscher Chemiker, Acta Praehistorica et Archaeologica 21, 1989, 9-39.

STARAC 1991

R. Starac, Prilog poznavanju keramike brončanog doba na Kvarneru. Unpublizierte Diplomarbeit, Filozofski fakultet Sveučilište u Zagrebu, Zagreb 1991.

STUCHLÍK 2003

S. STUснцíк, Die Beziehungen Mährens zu den südöstlichen Gebieten am Anfang der Bronzezeit. In: C. Kacsó (Hrsg.), Bronzezeitliche Kulturerscheinungen im karpatischen Raum: Die Beziehungen zu den benachbarten Gebieten. Ehrensymposium für Alexandru Vulpe zum 70. Geburtstag, Baia Mare, 10.-13. Oktober 2001. Baia Mare 2001, 445-468.

TeržAn, Mihovilić, HäNSEl 1999

B. TeržAn, K. Mihovilić, B. Hänsel, Eine protourbane Siedlung der älteren Bronzezeit im istrischen Karst, Prähistorische Zeitschrift 74, 1999, 154-193.

Tiefengraber 2018

G. Tiefengraber, Der Wildoner Schlossberg: Die Ausgrabungen des Landesmuseums Joanneum 1985-1988. Schild von Steier, Beiheft 7, Forschungen zur geschichtlichen Landeskunde der Steiermark 80, Graz 2018.

URANKAR 2020

R. URANKAR, Kemijske analize brončanodobnih metalnih predmeta iz Monkodonje / Chemische Analysen der bronzezeitlichen Metallfunde aus Monkodonja. In: B. Hänsel, K. Mihovilić, B. TeržAn, Monkodonja: Istraživanje protourbanog naselja brončanog doba Istre 3: Nalazi od metala, gline, kosti i kamena kao i ljudskih i životinjskih kostiju / Monkodonja: Forschungen zu einer protourbanen Siedlung der Bronzezeit Istriens 3: Die Funde aus Metall, Ton, Knochen und Stein sowie die menschlichen und tierischen Knochen. Monografije i katalozi Arheološki muzej Istre 34, Pula 2020, 251-262.

URBAN 1993

T. URBAn, Studien zur mittleren Bronzezeit in Norditalien. Universitätsforschungen zur prähistorischen Archäologie 14, Bonn 1993.

WeMHofF, Rind 2018

M. Wemhoff, M. M. Rind (Hrsg.), Bewegte Zeiten: Archäologie in Deutschland. Petersberg 2018.
ZICH 1996

B. ZICH, Studien zur regionalen und chronologischen Gliederung der nördlichen Aunjetitzer Kultur. Vorgeschichtliche Forschungen 20, Berlin - New York 1996.

ZICH 2004

B. Zich, Das Ende der Aunjetitzer Kultur. In: H. Meller (Hrsg.), Der geschmiedete Himmel: Die weite Welt im Herzen Europas vor 3600 Jahren. Stuttgart 2004, 136-137.

ZuPANČIČ et al. 2012

N. Zupančič, S. Skobe, M. Miler, K. Mihovilić, B. Hänsel, B. TeržAn, Porijeklo ranobrončanodobnih sjekira od zelenog kamena iz Monkodonje / Provenance of the early bronze age greenstone axes from Monkodonja, Histria archaeologica 43, 2012, 5-17.

Anja Hellmuth Kramberger Alma Mater Europaea Institutum Studiorum Humanitatis Fakulteta za podiplomski bumanistični študij Ljubljana Kardeljeva ploščad 1 1000 Ljubljana Slowenien agratb@web.de orcid.org/0000-0002-8084-2771 